Research Articles: Behavioral/Cognitive

\title{
Subregions of DLPFC display graded yet distinct structural and functional connectivity
}

https://doi.org/10.1523/JNEUROSCI.1216-21.2022

Cite as: J. Neurosci 2022; 10.1523/JNEUROSCI.1216-21.2022

Received: 14 June 2021

Revised: 10 November 2021

Accepted: 2 January 2022

This Early Release article has been peer-reviewed and accepted, but has not been through the composition and copyediting processes. The final version may differ slightly in style or formatting and will contain links to any extended data.

Alerts: Sign up at www.jneurosci.org/alerts to receive customized email alerts when the fully formatted version of this article is published.

Copyright @ 2022 Jung et al.

This is an open-access article distributed under the terms of the Creative Commons Attribution 4.0 International license, which permits unrestricted use, distribution and reproduction in any medium provided that the original work is properly attributed. 
6

\title{
Subregions of DLPFC display graded yet distinct structural and
} functional connectivity

\author{
Abbreviated title: structural and functional connectivity of DLPFC
}

JeYoung JUnG ${ }^{1}$, Matthew A. LAmbon RALPH ${ }^{2}$ \& Rebecca L. JACKSON ${ }^{2}$

${ }^{1}$ School of Psychology, University of Nottingham, Nottingham NG7 2RD, UK

${ }^{2}$ MRC Cognition and Brain Sciences Unit, University of Cambridge, Cambridge CB2

7EF, UK

Corresponding authors:

Dr JeYoung Jung

School of Psychology University of Nottingham, University Park, Nottingham

NG7 2RD UK

Tel: +44(0) 1158467241

Email: jeyoung.jung@nottingham.ac.uk

Dr Rebecca L. Jackson

MRC Cognition and Brain Science Unit (CBU), University of Cambridge, Cambridge CB2 7EF UK

Tel: +44 (0) 1223769452

Email: rebecca.jackson@mrc-cbu.cam.ac.uk

\section{Conflict of interest}

The authors declare no competing financial interests.

\section{Acknowledgments}

This research was supported by a Beacon Anne McLaren Research Fellowship (University of Nottingham) to JJ, a British Academy Postdoctoral Fellowship awarded to RLJ (pf170068), and an Advanced ERC award (GAP: 670428 -

BRAIN2MIND_NEUROCOMP), MRC programme grant (MR/R023883/1), and intramural funding (MC_UU_00005/18) to MALR. 


\section{Abstract}

The human dorsolateral prefrontal cortex (DLPFC, approximately corresponding to Brodmann areas 9 and 46) has demonstrable roles in diverse executive functions such as working memory, cognitive flexibility, planning, inhibition, and abstract reasoning. However, it remains unclear whether this is the result of one functionally homogeneous region or whether there are functional subdivisions within the DLPFC. Here, we divided the DLPFC into seven areas along rostral-caudal and dorsalventral axes anatomically and explored their respective patterns of structural and functional connectivity. In vivo probabilistic tractography (11 females and 13 males) and resting-state functional magnetic resonance imaging (57 females and 21 males) were employed to map out the patterns of connectivity from each DLPFC subregion. Structural connectivity demonstrated graded intra-regional connectivity within the DLPFC. The patterns of structural connectivity between the DLPFC subregions and other cortical areas revealed that the dorsal-rostral subregions were restricted to connection to other frontal and limbic areas, whereas the ventral-caudal region was widely connected to frontal, parietal, and limbic cortex. Functional connectivity analyses demonstrated that subregions of DLPFC were strongly interconnected to each other. The dorsal subregions were associated with the default mode network $(\mathrm{DMN})$, while middle dorsal-rostral subregions were linked with the multiple demand network (MDN), respectively. The ventral-caudal subregion showed increased functional coupling with both DMN and MDN. Our results suggest that the connectivity of the DLPFC may be subdivided along a dorsal rostral - ventral caudal axis with differing (albeit graded) patterns of connectivity reflecting the integrative executive function of the DLPFC.

\section{Keywords}

Dorsolateral prefrontal cortex; tractography, resting-state fMRI, structural connectivity; functional connectivity; executive control

\section{Significance Statement}

Research has shown that the dorsolateral prefrontal cortex (DLPFC) plays a role in various executive functions. By dividing the DLPFC into seven areas along rostralcaudal and dorsal-ventral axes anatomically, we explored their patterns of structural and functional connectivity. The patterns of connectivity within DLPFC subregions demonstrated graded intra-regional connectivity. The patterns of connectivity between the DLPFC subregions and other cortical areas revealed distinctive patterns of connectivity from dorsal-rostral subregions to ventral-caudal subregions. Divisions across DLPFC subregions seem to align with their structural and functional connectivity. Our results suggest that DLPFC may be subdivided by the diagonal axis of the dorsal-ventral axis and rostral-caudal axis, supporting the framework of a functional organization along the anterior-posterior axis in the lateral prefrontal cortex. 


\section{Introduction}

The dorsolateral prefrontal cortex (DLPFC) approximately corresponds to Brodmann areas (BA) 9 and 46 and consists of the lateral part of superior frontal gyrus (SFG) and middle frontal gyrus (MFG) (Brodmann, 1908; Walker, 1940; Petrides and Pandya, 1999). More recently, Petrides (2005b) designated DLPFC as BA 9, BA 46, and BA 9/46. Previous anatomical and functional studies have demonstrated differences in the subparts of lateral prefrontal cortex connectivity including the DLPFC (For the review, see Petrides, 2005b; Thiebaut de Schotten et al., 2012; Cieslik et al., 2013). Although the DLPFC can be divided into two or three subregions cytoarchitectonically, the functional role of each subregion is not clear.

DLPFC plays an important role in executive functions, such as working memory, cognitive flexibility, planning, inhibition, and abstract reasoning (Miller and Cummings, 2007) and is connected to a variety of brain regions including the thalamus, basal ganglia, hippocampus, and associative cortex such as posterior temporal, and parietal areas (Petrides and Pandya, 1984; Morris et al., 1999; Petrides, 2005b; Yeterian et al., 2012). Anatomical studies have demonstrated that different areas within the DLPFC receive their input from distinct subparts of the parietal cortex (Petrides and Pandya, 1984; Thiebaut de Schotten et al., 2012) and that the functional role of the DLPFC may be partly determined by its anatomical connections to other brain regions (Morecraft et al., 2004; Hoshi, 2006). Strong evidence from studies with both human and primates suggests an anterior-posterior axis of functional organization of the lateral prefrontal cortex (Koechlin et al., 2003; Petrides, 2005b, a; Thiebaut de Schotten et al., 2012). Lesions in the caudal DLPFC (BA 46 and 9/46) are associated with a deficit on monitoring of information in working memory whereas additional caudal lesions to BA6 and 8 impair tasks requiring selection between alternative choices (Petrides, 1985, 2005a). The rostral mid-lateral prefrontal regions (BA 10 and 46) play a more abstract role in cognitive control (Petrides, 2005b; Moayedi et al., 2015). In addition to the caudal-rostral axis, there is a dorsal-ventral axis of organization of the mid-lateral prefrontal cortex (DLPFC: BA 46 and 9/46) (Petrides and Pandya, 1999; Petrides, 2005b). In monkeys, the dorsal DLPFC (BA9/46d) plays a role in motor planning, multi-tasking, and maintaining goals whereas the ventral DLPFC (BA9/46v) is preferentially involved in the visuospatial information of attended signals and cues. These findings suggest a possibility that some functionally distinct subparts may exist within the DLPFC.

Despite the well-documented role of the DLPFC in various executive functions, it remains unclear whether functional subdivisions are present within the DLPFC. DLPFC is activated for a range of cognitive demands such as response inhibition, working memory, and decision making as a key node of the multiple demand network (MDN) including the inferior frontal gyrus (IFG), frontal eye field, insular, presupplementary motor area (pre-SMA), anterior cingulate cortex (ACC), and intraparietal sulcus (IPS) (Duncan, 2010). Although numerous fMRI studies report DLPFC activation, the exact location and extent of activation sites vary according to 
tasks used in those studies (Nee et al., 2007; Zheng et al., 2008; Rottschy et al., 2012; Bahlmann et al., 2014; Kohn et al., 2014). One study parcellated the DLPFC using a meta-analysis of task-dependent and task-independent connectivity (Cieslik et al., 2013). They delineated the DLPFC into two regions with different connections: the anterior subregion co-activated with the anterior cingulate cortex (ACC) and the posterior subregion co-activated with the parietal cortex. These findings support functional variation along a rostral-caudal axis in the DLPFC (Petrides, 2005a). In addition, a dorsal-posterior region of DLPFC was associated with the default mode network (DMN), demonstrating functional connections with posterior cingulate cortex, ventromedial prefrontal cortex, and angular gyrus (AG) (Alves et al., 2019). Together, the functional heterogeneity and diversity of anatomical connections in the human DLPFC suggests it may consist of functionally distinct subregions.

Here, we test whether there are connectivity differences across the rostralcaudal and dorsal-ventral axes in the DLPFC that would lead to different functional subregions. We used diffusion weighted imaging (DWI) and resting state fMRI to explore the structural and functional connectivity across the DLPFC in human participants. To improve signal in ventromedial frontal and anterior temporal regions, while maintaining signal across the whole brain, we employed probabilistic tractography of distortion-corrected DWI (Embleton et al., 2010) and seed-based analysis of dual-echo resting state fMRI (Halai et al., 2014). The DLPFC was separated into seven different areas based on the rostral-caudal and dorsal-ventral divisions of the Brodmann regions (BA9, 46, and 9/46) (Petrides, 2005b). For each seed region, we investigated and compared the structural connectivity to sixty-three target regions including left frontal, temporal, parietal, and limbic cortex and the whole brain functional connectivity. We hypothesized that there would be functional subregions of the DLPFC determined by their structural or functional connectivity and that there would be differential patterns of structural and functional connectivity within the DLPFC along the rostral-caudal or dorsal-ventral axis.

\section{Materials and Methods}

\section{Subjects and data acquisition}

Two different datasets were employed in this study. Dataset 1 included 24 healthy, right-handed subjects (11 females; mean age $=25.9$, range 19-47), whereas dataset 2 included 78 healthy right-handed subjects ( 57 females; means age $=25.2$, range 20-44). Each dataset has been utilized for various investigations: Dataset 1 (Cloutman et al., 2012; Bajada et al., 2016; Bajada et al., 2017; Jung et al., 2017; Jackson et al., 2020). Dataset 2 (Jackson et al., 2016; Jackson et al., 2018; Jung et al., 2018; Jackson et al., 2020). Dataset 1 consisted of distortion-corrected DWI and structural MR imaging. DWI was performed using a pulsed gradient spin echo-planar sequence, with TE $=59 \mathrm{~ms}, T R \approx 11884 \mathrm{~ms}, \mathrm{G}=62 \mathrm{mTm}^{-1}$, half scan factor $=0.679$, $112 \times 112$ image matrix reconstructed to $128 \times 128$ using zero padding, reconstructed resolution $1.875 \times 1.875 \mathrm{~mm}$, slice thickness $2.1 \mathrm{~mm}, 60$ contiguous 
slices, 61 non-collinear diffusion sensitization directions at $b=1200 \mathrm{smm}^{-2}(\Delta=29.8$ $\mathrm{ms}, \delta=13.1 \mathrm{~ms}), 1 \mathrm{at} b=0$, SENSE acceleration factor $=2.5$. Acquisitions were cardiac gated using a peripheral pulse unit positioned over the participants' index finger or an electrocardiograph. For each gradient direction, two separate volumes were obtained with opposite polarity $k$-space traversal with phase encoding in the left-right/right-left direction to be used in the signal distortion correction procedure (Embleton et al., 2010). A co-localized T2 weighted turbo spin echo scan, with inplane resolution of $0.94 \times 0.94 \mathrm{~mm}$ and slice thickness $2.1 \mathrm{~mm}$, was obtained as a structural reference scan to provide a qualitative indication of distortion correction accuracy. A high resolution T1-weighted 3D turbo field echo inversion recovery image $(\mathrm{TR} \approx 2000 \mathrm{~ms}, \mathrm{TE}=3.9 \mathrm{~ms}, \mathrm{TI}=1150 \mathrm{~ms}$, flip angle 8ㅇ, $256 \times 205$ matrix reconstructed to $256 \times 256$, reconstructed resolution $0.938 \times 0.938 \mathrm{~mm}$, slice thickness $0.9 \mathrm{~mm}, 160$ slices, SENSE factor $=2.5$ ) was used.

Dataset 2 included resting-state fMRI and structural MR imaging. To cover the whole brain without signal dropout around the rostral temporal and inferior frontal cortices, a dual-echo fMRI protocol was performed (Poser et al., 2006; Halai et al., 2014). This involves parallel acquisition at a short echo $(12 \mathrm{~ms})$ leading to less signal loss in areas of high magnetic susceptibility and a standard long echo (35ms) to maintain high contrast sensitivity throughout the brain. The results from the two echoes were combined using linear summation, previously shown to be optimal. The fMRI parameters included 42 slices, 80 × 80 matrix, $240 \times 240 \times 126 \mathrm{~mm}$ FOV, inplane resolution $3 \times 3$, slice thickness $4 \mathrm{~mm}$. 130 volumes were collected over 6.25 minutes. T1-weighted structural images were acquired using a 3D MPRAGE pulse sequence with 200 slices, in-planed resolution $0.94 \times 0.94 \mathrm{~m}$ slice thickness $1.2 \mathrm{~mm}$, $\mathrm{TR}=8.4 \mathrm{~ms}, \mathrm{TE}=3.9 \mathrm{~ms}$. During resting-state $\mathrm{fMRI}$, all subjects were instructed to keep their eyes open and look at the fixation cross. Imaging data were acquired on a 3T Philips Achieva scanner (Philips Medical System, Best Netherlands). The study was approved by the local ethics committee and all participants provided written informed consent forms.

\section{Definition of seed regions and target masks}

In order to divide the DLPFC (BA 9 and BA46) on the rostral-caudal dimension and to explore differences in DLPFC connectivity, seven anatomically defined regions of interest (ROIs) were defined in the left hemisphere (Fig.1A): two located on BA 9 (anterior: 9a, posterior: 9p), two placed in dorsal-middle frontal gyrus (anterior: 9/46da, posterior: 9/46dp), one was on BA 46 (46), and two placed in ventral-middle frontal gyrus (anterior: 9/46va, posterior: 9/46vp). The seeds regions were identified as a sphere with $6 \mathrm{~mm}$ radius in the MNI template brain based on topographic description and defined carefully without overlapping each other. 63 target regions covering frontal, temporal, parietal, and limbic cortex were defined using WFU Pick Atlas (Maldjian et al., 2003) and SPM Anatomy toolbox (Eickhoff et al., 2005). It should be noted that the occipital lobe was not included in this study 
because there was no direct white matter pathways connecting the DLPFC and occipital lobe (Petrides and Pandya, 1984; Morris et al., 1999; Petrides, 2005b; Yeterian et al., 2012). The frontal lobe regions included BA 10 (frontal pole: FP), BA 44 (pars opercularis), BA 45 (pars triangularis), BA47 (pars orbitalis), medial orbitofrontal cortex (medOFC), lateral OFC (latOFC), supplementary motor area (SMA), and primary motor cortex (M1). The parietal lobe had 7 regions in superior parietal cortex (SPC: 5L, 5M, 5Ci, 7A, 7PC, 7M, 7P), 3 regions in intraparietal sulcus (IPS: IPS1, IPS2, IPS3), and 7 regions in inferior parietal cortex (IPC: PFop, PFt, PF, PFm, PFcm, PGa, PGp). The temporal lobe had 20 ROls covering superior temporal gyrus (STG), middle temporal gyrus (MTG), inferior temporal gyrus (ITG), fusiform gyrus (FG), parahippocampal gyrus (PhG) (please, see the detailed location of the temporal ROIs for Jung et al., 2017; Jung et al., 2018). The limbic lobe included insular, amygdala, hippocampus, caudate, putamen, pallidum, thalamus, and 3 regions of cingulate cortex (anterior cingulate cortex; ACC, middle CC; MCC, posterior CC; PCC). The DARTEL (diffeomorphic anatomical registration through an exponentiated lie algebra) toolbox (Ashburner, 2007) was used to transform the seeds and masks from the MNI space into each individual's native diffusion space. The transform was estimated using each subject's T1-weighted image coregistered to their diffusion weighted images. The accuracy of the transformation of ROIs into native space was inspected using the anatomical images. For resting-state functional connectivity analysis, ROls without DARTEL transformations were used, as analysis was performed in MNI space.

\section{Probabilistic tractography}

Unconstrained probabilistic tractography was performed using the PICo software package (Parker and Alexander, 2005), sampling the orientation of probability density functions (PDFs) which was generated using constrained spherical deconvolution (Tournier et al., 2008) and model-based residual bootstrapping (Haroon et al., 2009; Jeurissen et al., 2011). 20,000 Monte Carlo streamlines were initiated from each voxel in the DLPFC seed regions. Step size was set to $0.5 \mathrm{~mm}$. Stopping criteria for the streamlines included terminating if the pathway curvature over a voxel was greater than $180^{\circ}$, or the streamline reached a physical path limit of $500 \mathrm{~mm}$. A single whole-brain probabilistic map was generated for each of the 7 seed ROIs for each participant. Probability maps were masked with each ROI and the maximum connectivity value (ranging from 0 to 20,000) between the seeds and each mask was extracted. The resultant connectivity matrices were subjected to a double threshold to ensure that only connections with high probability in the majority of participants were considered. For the first-level individual threshold, following the approach described by Cloutman et al. (2012), the $\lambda$-value of the Poisson distribution identified was used to determine a threshold value at $p=0.025$. For the second-level group threshold, we used two criteria for consistency (over $75 \%$ 
of participants, i.e., at least $18 / 24$ participants and over $50 \%$ of participants, i.e., at least 12/24 participants).

\section{Resting-state fMRI data analysis}

Pre-processing was performed using SPM 8 and the data processing assistant for Resting State fMRI (DPARSF Advanced Edition, V2.3) toolbox. The first two volumes were discarded to allow for magnetic saturation effects. The images were slice-time corrected, realigned, and coregistered to the individual's structural image using SPM 8. Censoring was applied using a threshold of greater than $3 \mathrm{~mm}$ of translation or 1 degree of rotation, which resulted in the exclusion of 6 participants from further analysis. Within DPARSF nuisance covariates were regressed out and the images were normalised using DARTEL, smoothed with an $8 \mathrm{~mm}$ full-width half maximum (FWHM) Gaussian kernel. The results were filtered at $.01-.08 \mathrm{~Hz}$. Nuisance covariates were regressed out including 24 motion parameters calculated from the 6 original motion parameters using Volterra expansion (Friston et al., 1996), which was shown to improve motion correction compared to the 6 parameters alone (Yan et al., 2013; Power et al., 2014). Additional covariates were included for outlier time points with a with a z-score greater than 2.5 from the mean global power or more than $1 \mathrm{~mm}$ translation as identified using the ARtifact detection Tools software package (ART; www.nitrc.org/projects/artifact_detect). These were entered as covariates with white matter, cerebrospinal fluid (CSF) and global tissue signal. Then, linear detrending was performed. Seed-based functional connectivity analyses were performed using DPARSF. Functional connectivity maps from the seeds were zscore normalised. One sample t-tests were used to detect areas with significant connectivity to the seed regions. The resultant images were thresholded at $p<0.001$, FWE-corrected at the cluster level. Comparisons between the functional connectivity maps of different seed regions were conducted using paired t-tests.

\section{Similarity analysis}

To provide formal spatial inference on the both the comparisons between subregions of DLPFC, we computed similarity of the connectivity patterns of DLFPC subregions. Using the connectivity patterns of DLPFC subregions, we constructed a similarity matrix for the structural and functional connectivity by conducting correlation analyses between each DLPFC subregion's connectivity patterns. In the similarity matrix, the patterns of connectivity between each DLPFC subregion and other brain areas were similar - similarity ' 1 ', whereas that of the DLPFC subregions was dissimilar - similarity ' 0 '. Then, we performed one-sample t-test for similarity matrix to determine a significant similarity between DLPFC subregions.

\section{Results}


Structural connectivity patterns across the DLPFC

Using probabilistic tractography, the structural connectivity for each DLPFC seed was identified, see Table 1 and Fig. 1. The full pattern of connectivity across the brain may be seen in Fig. 2. There was strong intra-DLPFC connectivity on the dorsal-ventral axis (along the gyri). Additionally, the mid-DLPFC regions (9/46da and 9/46dp) showed the strongest intra-DLPFC connectivity, with connections to more dorsal and ventral areas (Fig. 1B). Across the DLPFC, there was a high level of connectivity with limbic regions, especially the insular and basal ganglia (caudate, putamen, and pallidum) (Fig. 1C). The ventral-caudal seeds (9/46dp, 9/46va, and 9/46vp) showed structural connections with the thalamus. Only 9/46vp had a connection to hippocampus. However, no direct connection was identified between any seed regions and the amygdala. Fig. 1D shows the pattern of connectivity between the DLPFC seed regions and other lateral associative cortices. Most DLPFC seed regions showed strong connectivity with the frontal pole (FP) and inferior frontal gyrus (IFG: BA44 and BA45) but not the most ventral aspects of the prefrontal cortex, including pars orbitalis (BA47) and the OFC. Only the ventralcaudal seeds (9/46va and 9/46vp) had strong evidence of connections to primary and supplementary motor regions. Additionally, only the 9/46vp seed connects to somatosensory and dorsal parietal regions (7PC and IPS). It should be noted that the DLPFC seed regions did not show any connection with the temporal and inferior parietal cortices. Overall, all DLPFC seeds showed strong connectivity with the FP, IFG, and the limbic system. The tractography results suggest a single axis of changing connectivity from ventral-caudal to dorsal-rostral regions, with the key differences being between the most ventral-caudal regions and elsewhere. Specifically, the ventral-caudal seeds (9/46va and 9/46vp) show widespread structural connectivity to frontal, limbic, sensorimotor, and superior parietal cortex.

\begin{tabular}{ccccccccc}
\hline & & $9 a$ & $9 p$ & $9 / 46 \mathrm{da}$ & $9 / 46 \mathrm{dp}$ & 46 & $9 / 46 \mathrm{va}$ & $9 / 46 \mathrm{vp}$ \\
\hline DLPFC & $9 \mathrm{a}$ & $\mathbf{1 0 0}$ & $\mathbf{8 8}$ & 21 & 8 & 8 & 8 & 0 \\
& $9 \mathrm{p}$ & & $\mathbf{1 0 0}$ & $\mathbf{6 7}$ & $\mathbf{5 8}$ & 33 & 38 & 0 \\
& $9 / 46 \mathrm{da}$ & & & $\mathbf{1 0 0}$ & $\mathbf{1 0 0}$ & $\mathbf{1 0 0}$ & $\mathbf{6 7}$ & 4 \\
& $9 / 46 \mathrm{dp}$ & & & & 100 & 96 & $\mathbf{9 6}$ & 8 \\
& 46 & & & & & 100 & 96 & 13 \\
& $9 / 46 \mathrm{va}$ & & & & & & 100 & 75 \\
& $9 / 46 \mathrm{vp}$ & & & & & & & 100 \\
\hline Frontal & FP & 92 & $\mathbf{9 6}$ & $\mathbf{9 6}$ & $\mathbf{9 6}$ & $\mathbf{9 6}$ & $\mathbf{8 8}$ & 25 \\
& BA44 & 0 & 17 & 4 & 25 & 21 & $\mathbf{8 8}$ & $\mathbf{1 0 0}$ \\
& BA45 & 17 & $\mathbf{6 7}$ & 17 & $\mathbf{6 3}$ & $\mathbf{7 5}$ & $\mathbf{9 6}$ & $\mathbf{9 2}$
\end{tabular}




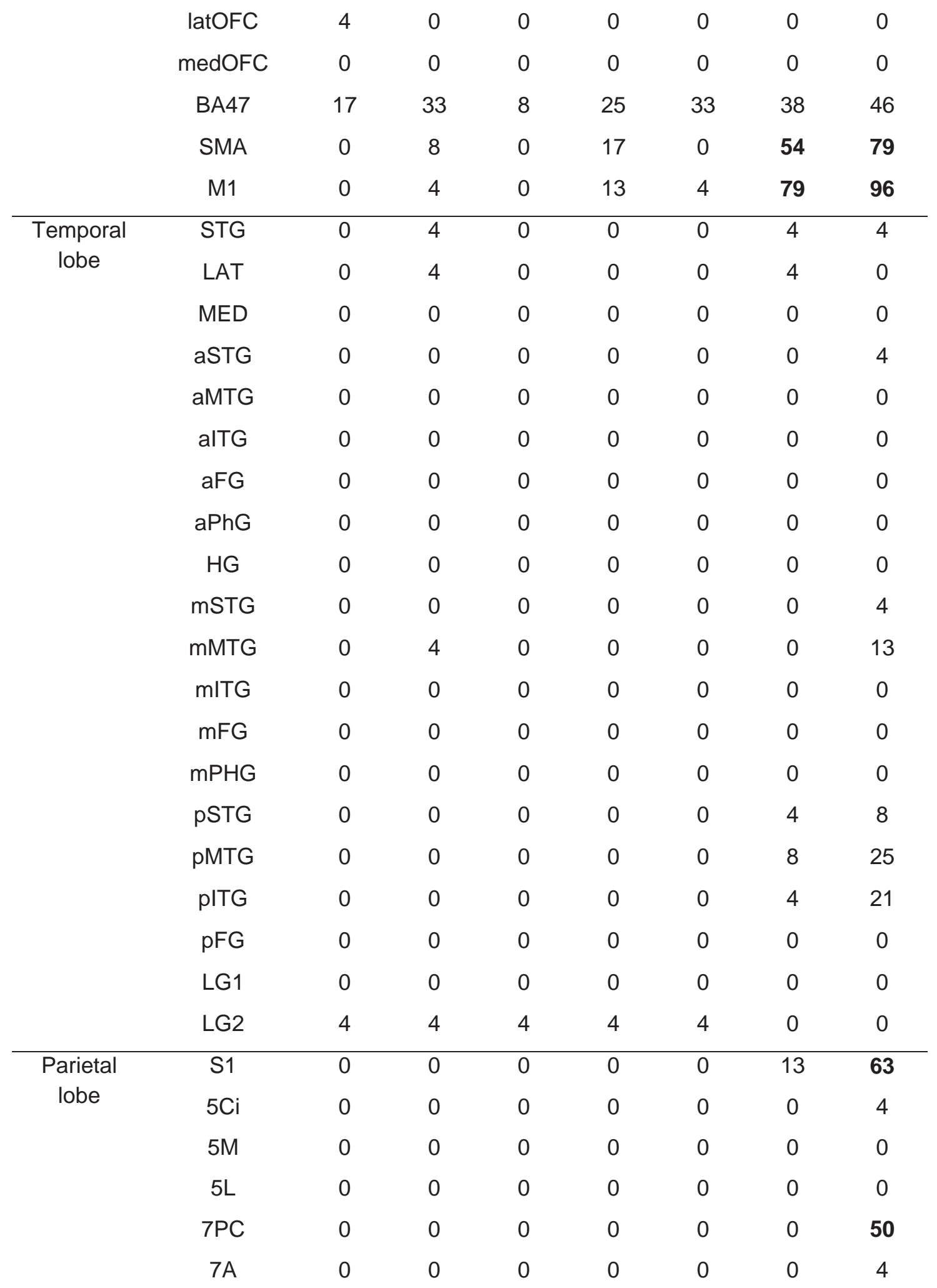




\begin{tabular}{|c|c|c|c|c|c|c|c|c|}
\hline & $7 P$ & 0 & 0 & 0 & 0 & 0 & 0 & 0 \\
\hline & $7 \mathrm{M}$ & 0 & 0 & 0 & 0 & 0 & 0 & 0 \\
\hline & IPS1 & 0 & 0 & 0 & 0 & 0 & 0 & 50 \\
\hline & IPS2 & 0 & 0 & 0 & 0 & 0 & 0 & 50 \\
\hline & IPS3 & 0 & 4 & 4 & 4 & 4 & 4 & 33 \\
\hline & PFo & 0 & 0 & 0 & 0 & 0 & 0 & 8 \\
\hline & PFt & 0 & 0 & 0 & 0 & 0 & 0 & 17 \\
\hline & PF & 0 & 0 & 0 & 0 & 0 & 0 & 25 \\
\hline & PFm & 0 & 0 & 0 & 0 & 0 & 0 & 17 \\
\hline & PFcm & 0 & 0 & 0 & 0 & 4 & 0 & 46 \\
\hline & PGa & 0 & 0 & 0 & 0 & 0 & 0 & 25 \\
\hline & PGp & 0 & 0 & 0 & 0 & 0 & 0 & 13 \\
\hline Limbic lobe & $A C C$ & 4 & 33 & 17 & 33 & 25 & 25 & 0 \\
\hline & MCC & 0 & 0 & 0 & 0 & 0 & 4 & 4 \\
\hline & PCC & 0 & 4 & 0 & 4 & 0 & 4 & 0 \\
\hline & Insular & 92 & 100 & 71 & 96 & 79 & 100 & 92 \\
\hline & caudate & 75 & 92 & 67 & 79 & 79 & 92 & 58 \\
\hline & putamen & 54 & 88 & 46 & 88 & 50 & 92 & 88 \\
\hline & pallidum & 42 & 83 & 38 & 67 & 42 & 88 & 88 \\
\hline & thalamus & 17 & 46 & 29 & 54 & 38 & 79 & 75 \\
\hline & amygdala & 0 & 0 & 0 & 0 & 0 & 0 & 0 \\
\hline & hippocampus & 13 & 13 & 4 & 4 & 4 & 29 & 63 \\
\hline
\end{tabular}

Table 1 Structural connectivity results for each DLPFC region. Bold font indicates that the connection probability was over 50\% (12/24) for group analysis.

$310 \mathrm{FP}=$ frontal pole; $\mathrm{BA}=$ Brodmann's areas; medOFC = medial orbitofrontal cortex;

311 latOFC= lateral orbitofrontal cortex; SMA=supplementary motor area; $\mathrm{M} 1$ = primary motor cortex; $\mathrm{S} 1$ = primary somatosensory cortex; IPS =intraparietal sulcus; $5 \mathrm{Ci}, 5 \mathrm{M}$, $5 \mathrm{~L}=\mathrm{BA} 5$ (superior parietal cortex); 7PC, 7A, 7P, 7M = BA 7 (superior parietal cortex); PFop, PFt, PF, PFcm, PFm = supramarginal gyrus; PGa, PGp = angular gyrus; $\mathrm{STG}=$ superior temporal gyrus; $\mathrm{LAT}=$ lateral temporal pole; $\mathrm{MED}=$ medial temporal pole; MTG = middle temporal gyrus; ITG = inferior temporal gyrus; FG = fusiform gyrus; $\mathrm{PhG}=$ parahippocampal gyrus; $\mathrm{HG}=$ Heschl's gyrus; $L \mathrm{G} 1$ = lingual gyrus next to fusiform gyrus; $L G 2=$ medial lingual gyrus; $A C C=$ anterior cingulate cortex; $\mathrm{MCC}=$ middle cingulate cortex $; \mathrm{PCC}=$ posterior cingulate cortex 


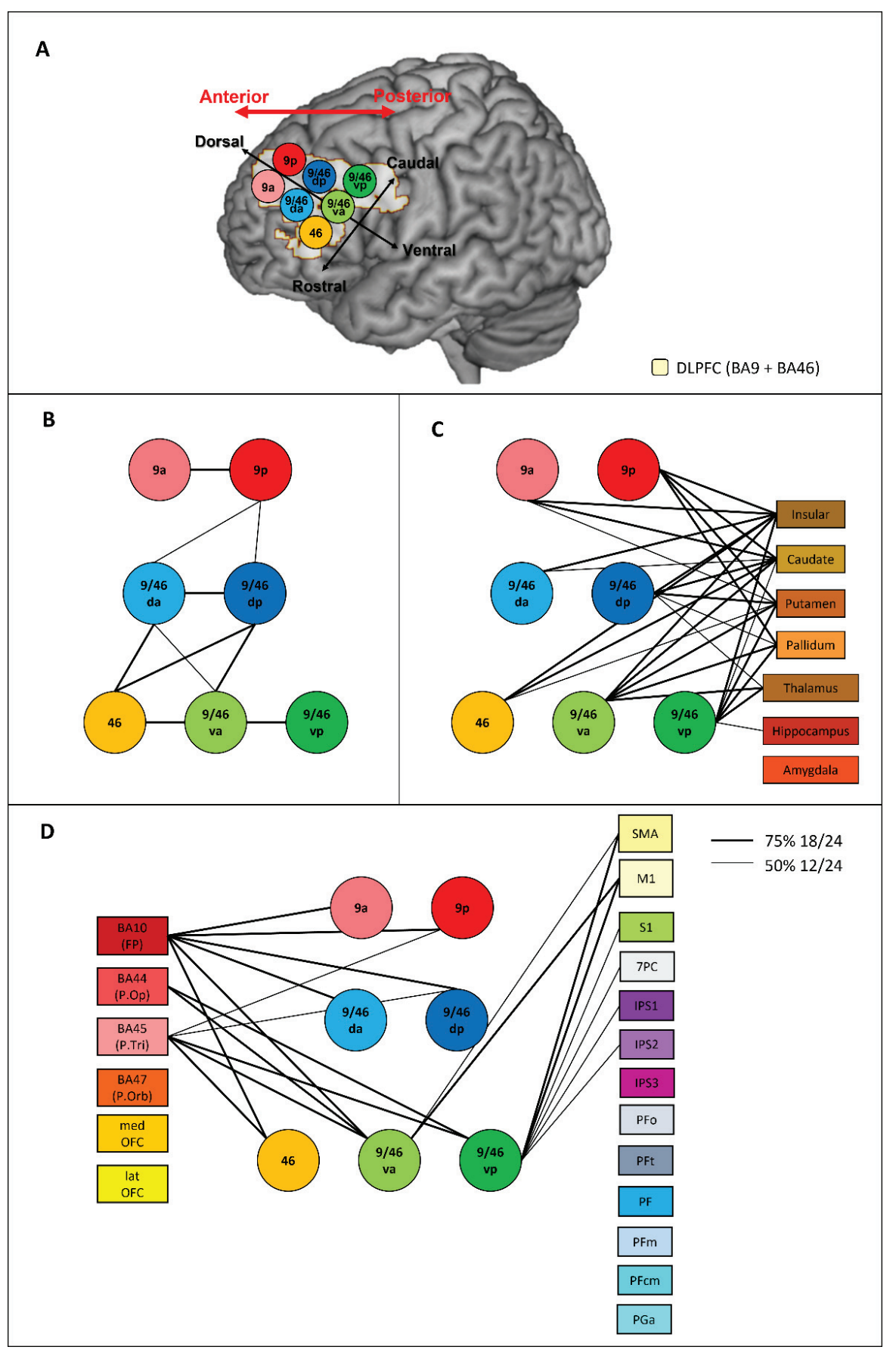

Figure 1. (A) The location of the seven DLPFC areas used as seed regions for the connectivity analyses. Red arrow indicates the anterior-posterior axis of the lateral prefrontal cortex. Black arrows represent each axis of the subregions of DLPFC. Brodmann area: BA; 9a: the anterior seed of BA9; 9p: the posterior seed of BA9; 9/46da: the dorsal-anterior seed of BA9/46; 9/46da: the dorsal-posterior seed of BA9/46; 46: BA46, 9/46va: the ventral-anterior seed of BA9/46; 9/46vp: the ventral- 
posterior seed of BA9/46. (B) Intra-DLPFC structural connectivity. (C) The structural connectivity between DLPFC seed regions and the limbic regions. (D) The structural connectivity between DLPFC seed regions and the frontal and parietal regions. Each DLPFC seed is represented by a circle. Lines connecting ROIs are displayed if the probabilistic tractography exceed the minimum probability threshold in either $50 \%$ (thin line) or $75 \%$ (thick line) of the participants.

$\mathrm{FP}=$ frontal pole; medOFC $=$ medial orbitofrontal cortex; latOFC $=$ lateral orbitofrontal cortex; SMA = supplementary motor area; $\mathrm{M1}$ = primary motor cortex; $\mathrm{S} 1$ = primary somatosensory cortex; 7PC = BA 7 (superior parietal cortex); IPS = inferior parietal sulcus; PFop, PFt, PF, PFcm, PFm = supramarginal gyrus; $\mathrm{PGa}=$ angular gyrus.

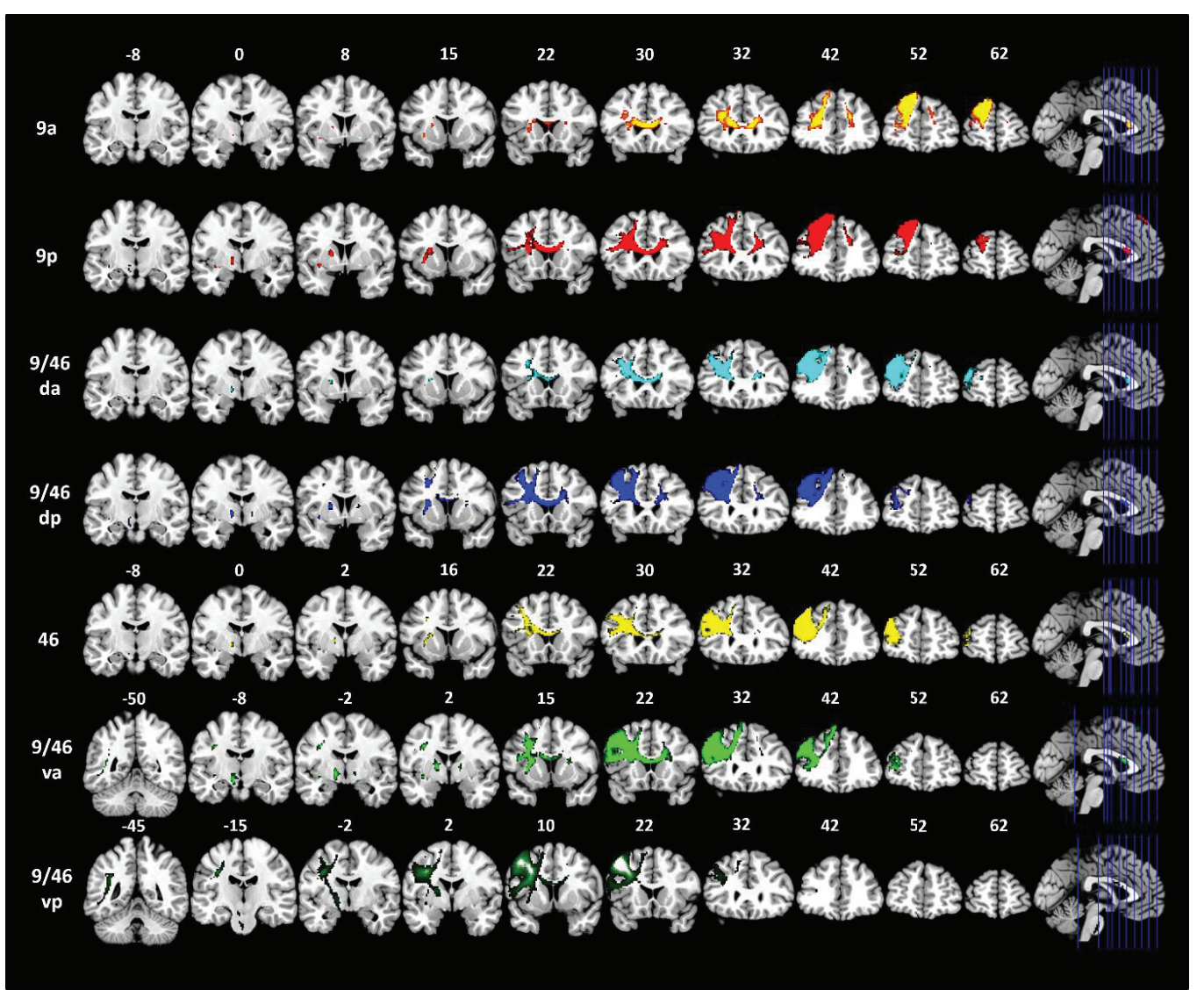

Figure 2. Structural connectivity patterns of the DLPFC seed regions.

Brodmann area: BA, 9a: the anterior seed of BA9, 9p: the posterior seed of BA9, 9/46da: the dorsal-anterior seed of BA9/46, 9/46da: the dorsal-posterior seed of BA9/46, 46: BA46, 9/46va: the ventral-anterior seed of BA9/46, 9/46vp: the ventralposterior seed of BA9/46. 


\section{Functional connectivity patterns across the DLPFC}

The whole-brain resting-state functional connectivity (rsFC) map of each DLPFC seed region is displayed in Fig. 3. Overall, the seven ROIs showed involvement in two distinct networks with gradedly different rSFC patterns suggesting a transition between these networks. The two BA 9 seeds (9a and 9p) were primarily correlated with the regions of the default mode network (DMN) including medial prefrontal cortex (mPFC), OFC, IPC (particularly angular gyrus), precuneus, PCC, anterior/middle temporal regions, and hippocampus (Raichle et al., 2001; Buckner et al., 2008). All other seed regions were strongly correlated with brain regions of the multiple demand network (MDN) including IFG, SMA, ACC/MCC, SPC, IPS, supramarginal gyrus, and pMTG (Duncan and Owen, 2000; Seeley et al., 2007; Woolgar et al., 2011; Spreng et al., 2013). However, area 9/46vp showed connectivity with both the MDN and the DMN. All DLPFC seed regions were strongly functionally connected to the insular and basal ganglia regions. The results appear to vary along the dorsal-ventral axis such that the dorsal parts of the DLPFC are connected with the DMN, whereas the ventral parts of DLPFC are associated with the MDN. Similar to the structural connectivity results, the most ventral-caudal seed (9/46vp) shows widespread functional connectivity across both of the DMN and MDN. 
stronger rsFC with the insula and IPL than 9p, whereas 9p showed higher rsFC with mPFC, angular gyrus, and precuneus than 9a. 9/46da showed higher rsFC with the IFG, insula, M1/S1, MCC, SPC, IPC, ITG, and visual cortex, yet lower rsFC with IPC, precuneus, PCC, and lateral temporal cortex than 9/46dp. The comparisons between the more rostral and caudal ventral seed regions exhibited prominent differences in similar regions. Relatively rostral regions showed higher $\mathrm{rSFC}$ with regions of the MDN including the IFG, SMA, M1/S1, supramarginal gyrus, ACC/MCC and visual cortex, yet lower rsFC with DMN regions, such as the MPFC, OFC, angular gyrus, precuneus, PCC, and lateral temporal cortex, than more caudal regions.

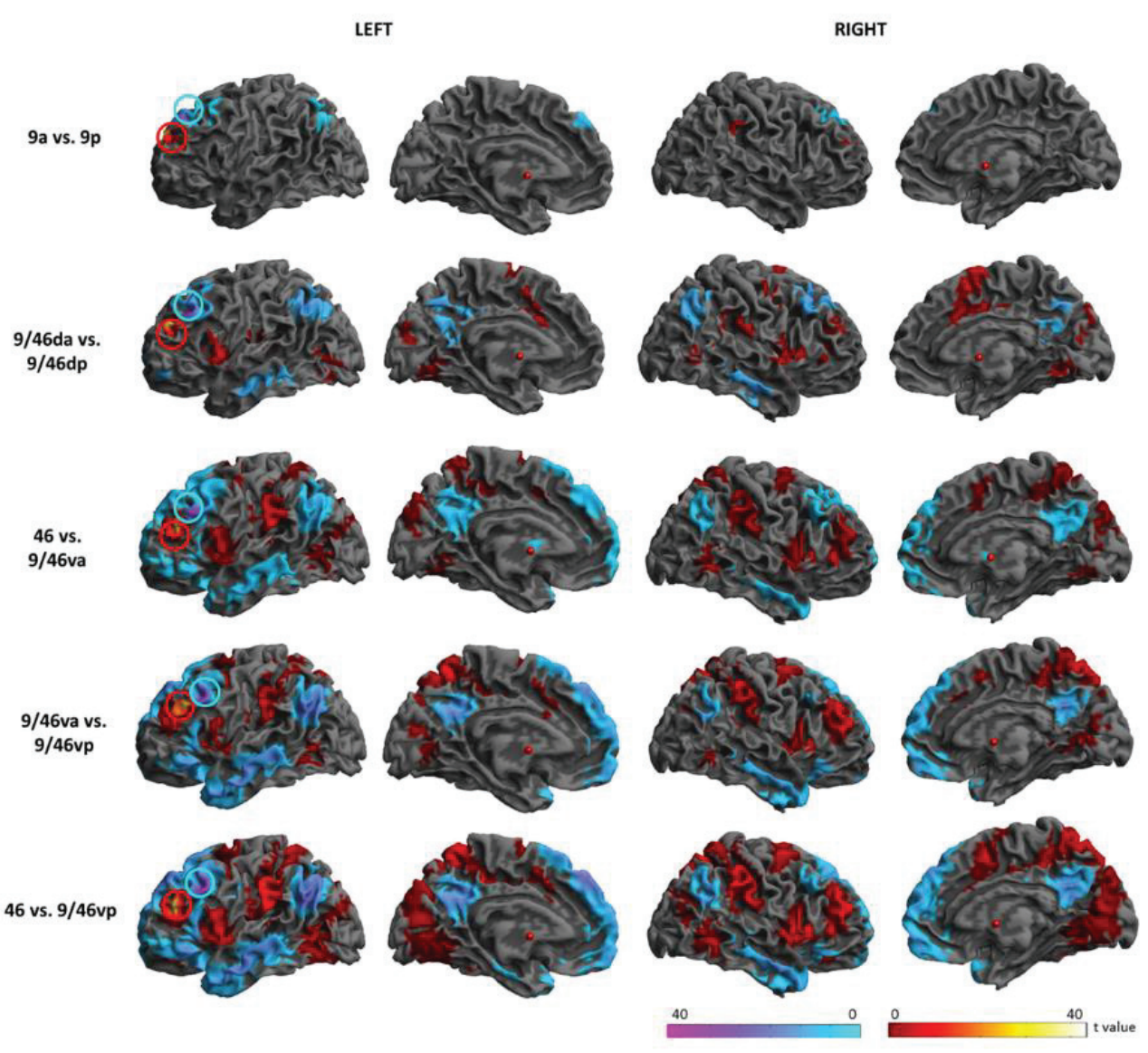

Figure 4. Comparison of the rsFC along the rostral-caudal axis. Circles indicate the DLPFC seed regions. Warm colours indicate the comparison from the rostral to the caudal regions. Cold colours indicate the comparison from the caudal to the rostral regions. Brodmann area: BA, 9a: the anterior seed of BA9, 9p: the posterior seed of BA9, 9/46da: the dorsal-anterior seed of BA9/46, 9/46da: the dorsal-posterior seed of BA9/46, 46: BA46, 9/46va: the ventral-anterior seed of BA9/46, 9/46vp: the ventral-posterior seed of BA9/46. 
In order to compute the differences between seed regions along the dorsalventral axis, we combined the each set of seed regions on the rostral-caudal axis. Fig. 5 shows the result of comparisons along the dorsal-ventral axis. Dorsal regions (9a and $9 p$ ) had significantly higher rsFC with the regions in the DMN and lower rsFC with the parts of the MDN than the middle regions (9/46da and 9/46dp). The middle regions showed higher rsFC with the MDN, yet lower rsFC with the DMN than ventral regions (46, 9/46va, and 9/46vp). The ventral regions had significantly higher rsFC with the MDN, yet lower rsFC with the DMN than the dorsal regions. Overall, the dorsal parts of the DLPFC had strong connectivity with the DMN, whereas the ventral DLPFC regions were strongly connected with the MDN.

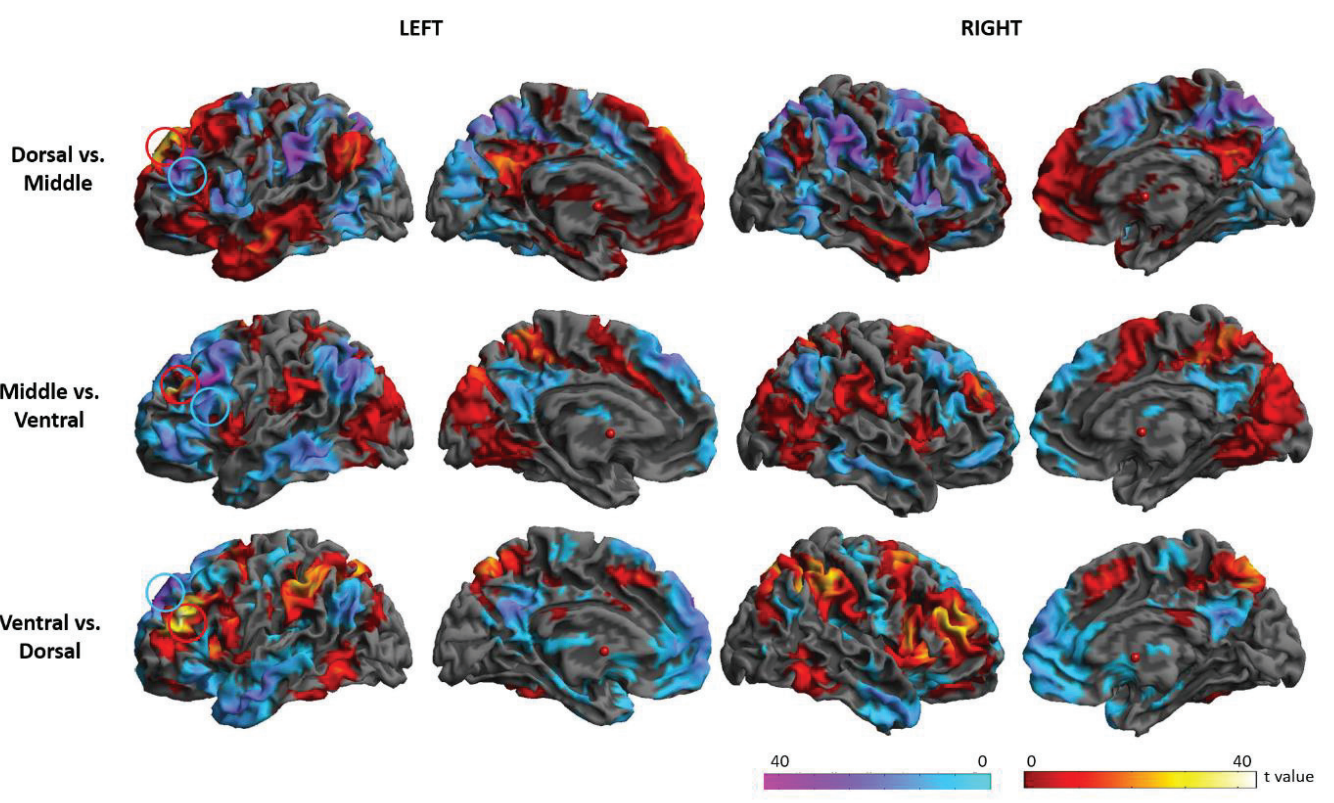

Figure 5. Comparison of the rsFC along the dorsal-ventral axis. Circles indicate the location of the DLPFC seed regions.

\section{Structural and functional connectivity profiles of DLPFC seed regions}

The connectivity profile of the DLPFC seed regions is displayed in Fig. 6A. Overall, the seed regions showed more widespread connections to target regions functionally than structurally (although the structural connectivity of 9/46vp was quite extensive), with structural connectivity mainly limited to the frontal and limbic cortex. With a more liberal threshold in structural connectivity (25\% of the participants), the dorsal- caudal seeds (9p and 9/46dp) and the ventral-rostral seed (46) showed a connection with the ACC and the most ventral-caudal seed (9/46vp) had a 
connection to the posterior MTG and angular gyrus (PGa) (Fig. 3 \& Fig. 6A).

Functional profiles of the DLPFC revealed the distinctive connectivity patterns of the BA 9 region was strongly coupled with the DMN (medial frontal lobe: latOFC \& medOFC; anterior temporal lobe: STG, LAT, MED, aSTG, \& aMTG; angular gyrus: $P G a \& P G p)$ and that of the BA 9/46vp was connected to the DMN (angular gyrus) as well as MDN (pMTG, pITG, \& IPS) (Fig. 6A). The other regions in middle and ventral DLPFC (BA 9/46da, 9/46dp, 46, and 9/46va) have strong functional connectivity with region in the MDN.

To compare the connectivity patterns of DLFPC regions, we used our ROIs and computed similarity of connectivity patterns according to each DLPFC region. The similarity matrix for structural and functional connectivity was constructed by computing the patterns of similarity (Pearson's correlation) (Fig. 6B). Then, we compared the similarity matrix between the DLPFC seed regions. The similarity results of structural connectivity revealed that most DLPFC subregions had similar patterns of connectivity such that the dorsal regions of DLPFC showed strong similarity to the ventral-anterior part of DLPFC (9/46va) and neighbouring regions along the rostral-caudal axis also revealed the very similar patterns of connectivity. However, 9/46vp did not showed such similarity with 9a, 9/46da, and 46. (Fig. 6C left). It demonstrated the distinctive patterns of structural connectivity between the most ventral-caudal region from dorsal-caudal regions (9a, 9/46da, and 46). Functional similarity of DLPFC subregions showed that (1) neighbouring regions showed similar patterns of connectivity and (2) the dorsal regions (9a and 9p) did not have functional similarity with the ventral regions (46 and 9/46va) (Fig. 6C right). Importantly, the ventral-caudal region showed the similar pattern of functional connectivity with all other DLPFC subregions, which is supported by its widespread structural connectivity. 

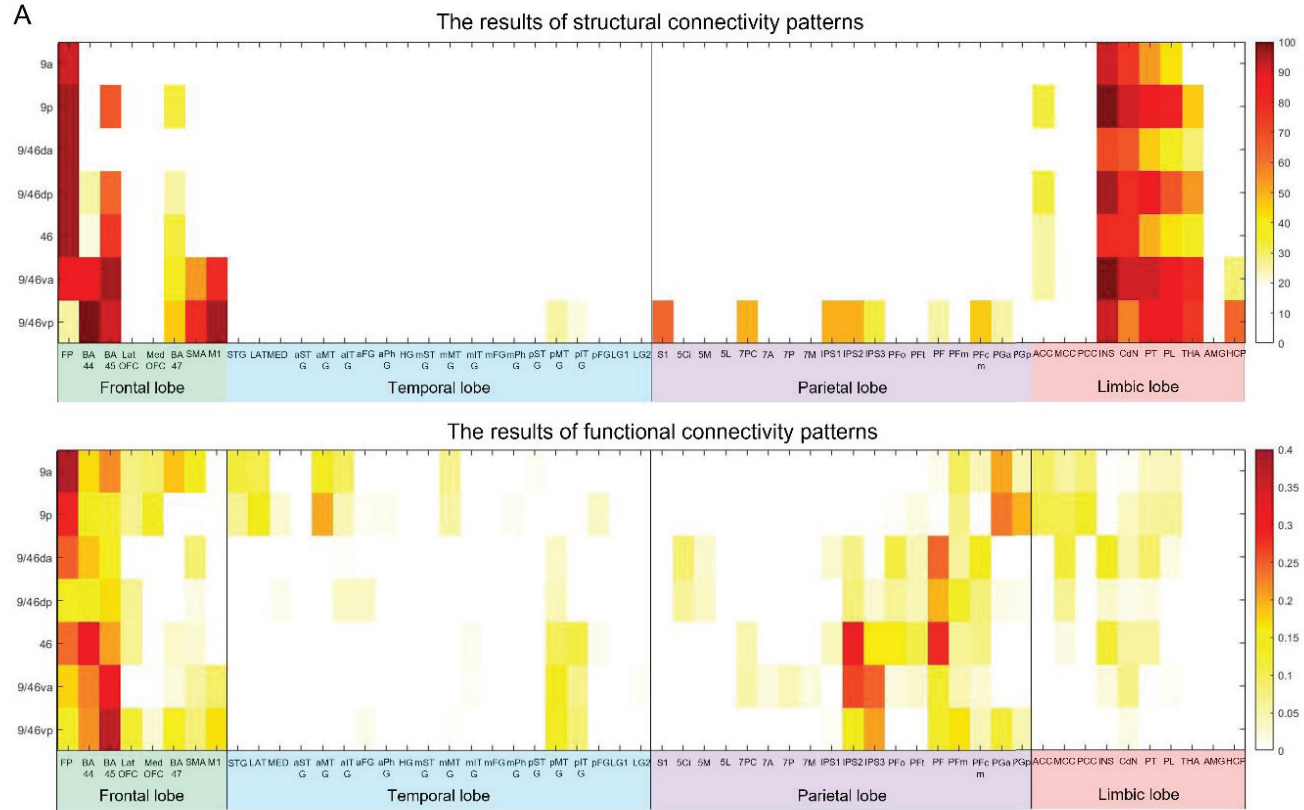

B
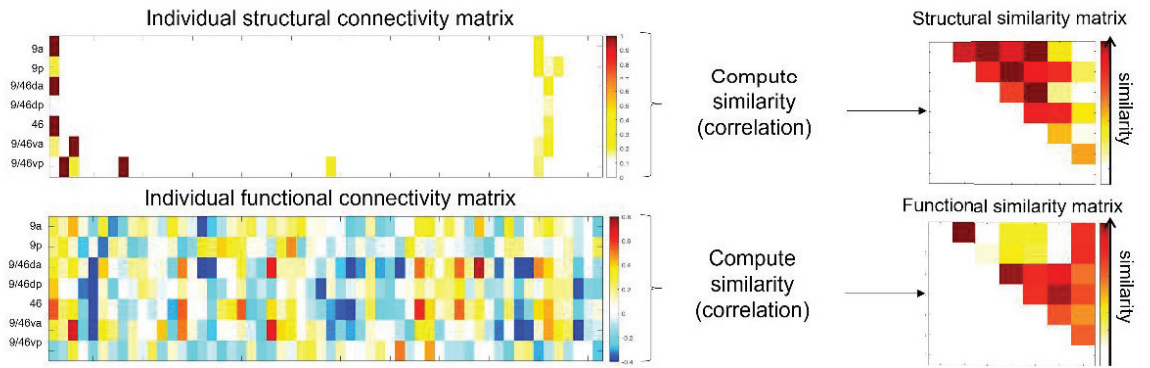

C

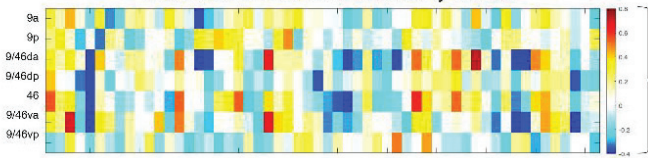

The similarity results of functional connectivity patterns
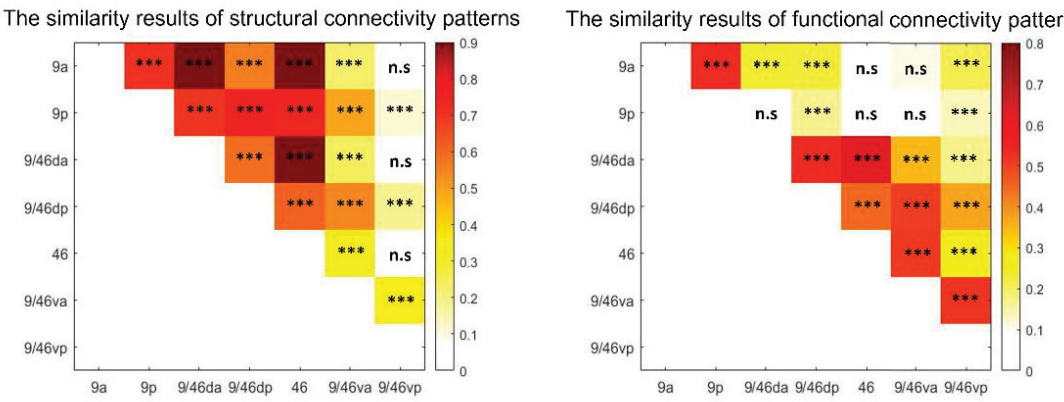

444

445

446

447

448

449

450

451

452
Figure 6. (A) The results of the structural (top) and functional (bottom) connectivity profiles of the DLPFC seed regions. The colour scale of structural connectivity indicates connection probability and that of functional connectivity indicates correlation coefficient. Negative correlations are not shown. (B) Similarity analysis. An example of structural and functional connectivity matrix from 7 DLPFC subregions. We computed the similarity for the patterns of connectivity of the DLPFC subregions. (C) The results of similarity analysis. ${ }^{* \star *} \mathrm{p}_{\text {Bonferroni-corrected }}<0.05(\mathrm{p}<$ 0.00023). 
$\mathrm{FP}=$ frontal pole; $\mathrm{BA}=$ Brodmann's areas; medOFC = medial orbitofrontal cortex; latOFC= lateral orbitofrontal cortex; SMA=supplementary motor area; $\mathrm{M} 1$ = primary motor cortex; $\mathrm{S} 1$ = primary somatosensory cortex; $5 \mathrm{Ci}, 5 \mathrm{M}, 5 \mathrm{~L}=\mathrm{BA} 5$ (superior parietal cortex); 7PC, 7A, 7P, 7M = BA 7 (superior parietal cortex); IPS = inferior parietal sulcus; PFop, PFt, PF, PFcm, PFm = supramarginal gyrus; PGa, PGp = angular gyrus; STG = superior temporal gyrus; LAT = lateral temporal pole; MED = medial temporal pole; MTG = middle temporal gyrus; ITG = inferior temporal gyrus; $F G$ = fusiform gyrus; PhG = parahippocampal gyrus; HG = Heschl's gyrus; LG1 = lingual gyrus next to fusiform gyrus; LG2 = medial lingual gyrus; $A C C=$ anterior cingulate cortex; $\mathrm{MCC}=$ middle cingulate cortex; $\mathrm{PCC}=$ posterior cingulate cortex; INS = insula; $\mathrm{CdN}=$ caudate nucleus; $\mathrm{PT}=$ putamen; $\mathrm{PL}=$ pallidum; $\mathrm{THA}=$ thalamus; $\mathrm{AMG}=$ amygdala; $\mathrm{HCP}=$ hippocampus

\section{Discussion}

We investigated the patterns of connectivity in subregions of DLPFC along the rostral-caudal and dorsal-ventral axes. We showed that subregions of DLPFC had differential structural and functional connectivity. Divisions across DLPFC subregions seem to align with their structural and functional connectivity. Structural connectivity analyses demonstrated graded intra-regional connectivity within the DLPFC. The patterns of connectivity between the DLPFC subregions and other cortical areas revealed a separation of dorsal-rostral subregions from the most ventral-caudal subregion. The dorsal-rostral subregions were restricted to link other frontal and limbic areas, whereas the ventral-caudal region was widely connected to frontal, temporal, parietal, and limbic cortex. The patterns of functional connectivity revealed that subregions of DLPFC were strongly interconnected to each other within the whole frontal cortex and coupled with two functional brain networks: MDN and DMN. The dorsal subregions were associated with the DMN, while middle dorsal-rostral subregions were linked with the MDN, respectively. Similar to the results of structural connectivity, the most ventral-caudal subregion showed increased functional coupling with both DMN and MDN. Our results suggest that DLPFC may be subdivided by the diagonal axis of the dorsal-ventral axis and rostral-caudal axis. Our findings support the framework of a functional organization along the anteriorposterior axis in the lateral prefrontal cortex (Petrides and Pandya, 1999; Koechlin et al., 2003).

The Cascade model proposes that executive control is implemented across the lateral prefrontal cortex along a posterior-to-anterior hierarchy, from simple to more abstract cognitive control processing (Koechlin et al., 2003). For example, posterior DLPFC supports action selection based on sensory input and anterior DLPFC provides episodic control for action selection, taking into account the ongoing context. The frontopolar cortex supports branching control for action selection based on a holding temporal context. Our structural connectivity results support this progressive posterior to anterior hierarchy within the DLPFC subregions, showing 
highly interconnected subregions within each gyri via short U-fibres, as well as DLPFC connections with the FP, IFG and motor regions via short frontal tracks (Catani et al., 2012; Yeterian et al., 2012). Specifically, the dorsal-rostral subregions connected to the FP via the frontal aslant tract, while the ventral-caudal subregions connect to sensory motor regions through the frontal longitudinal tracts. Similarly, our functional connectivity results demonstrated that subregions of DLPFC had strong coupling with other frontal regions including the FP, IFG, OFC, and motor cortex. The results of the intra-DLPFC connectivity were strong (especially between the neighbouring regions) and graded presumably reflecting local U-shaped fibres with few sharp divisions (9a was not structurally connected with other DLPFC seeds). The graded intra-DLPFC connectivity is consistent with primarily similar local computations, such that the region as a whole has the properties of mass action (Farah and McClelland, 1991). Furthermore, these patterns of connectivity within the DLPFC, along with connection to other frontal areas, suggest there is a graded and integrative organization of the whole frontal lobe (Petrides and Pandya, 1999; Petrides, 2005b).

We observed that the dorsal-rostral subregions (anterior parts of the DLPFC) were linked to the frontopolar regions with increased functional connectivity with the DMN. The frontopolar cortex is a supramodal area involved in various higher order functions such as self-directed thought, rational integration - the simultaneous consideration of multiple relations, and cognitive branching - holding goals while exploring secondary goals, planning, and reasoning (Ramnani and Owen, 2004). With co-activation of the frontopolar cortex, it has reported that the DMN could be activated for self-generated thought (Christoff et al., 2016) or increased cognitive reasoning complexity (Sormaz et al., 2018). These studies support our findings that dorsal-rostral subregions of the DLPFC connected with the FP, were strongly coupled with the DMN. In line with the anterior-posterior gradient in the prefrontal cortex, our connectivity analysis suggests that the anterior parts of the DLPFC would be involved in more challenging cognitive control, such as complex cognitive reasoning and cognitive branching.

In contrast, the middle-ventral subregions (middle-posterior parts) were connected to the IFG (BA 44 and 45) with strong coupling with the MDN and the ventral-caudal region (the most posterior part) had anatomical connections with temporal and parietal areas with increased functional connectivity with both DMN and MDN. Several cortico-cortical association pathways link the prefrontal cortex and other cortical regions (Petrides and Pandya, 1984; Petrides, 2005b; Catani et al., 2012; Thiebaut de Schotten et al., 2012; Yeterian et al., 2012). The superior longitudinal fasciculus (SLF) links the PFC and parietal cortex. SLF has three distinct branches: SLF I connecting the superior frontal area (BA 8, 9, 32) to SPC, SLF II connecting the SFG/MFG to IPS/AG, SLF III connecting IFG to IPS. The arcuate fasciculus (AF) connects the posterior regions of the frontal lobe and temporal lobe (Parker et al., 2005). The inferior fronto-occipital fasciculus (IFOF) connects occipital cortex, temporal areas, ventrolateral frontal cortex and inferior parietal regions 
(Schmahmann et al., 2007; Martino et al., 2010). As a part of the MDN, the IFG is involved in cognitive control and language processing (Brass et al., 2005; Camilleri et al., 2018). As the IPS shows anatomical connection with the DLPFC via SLF I/SLF II (Petrides and Pandya, 1984; Petrides, 2005a; Thiebaut de Schotten et al., 2012), the IPS acts as a multifaceted behavioural integrator, binding task-relevant information from the sensory, motor, and cognitive domains, mediated by the topdown control of DLPFC (Gottlieb, 2007). These findings suggest that the middleposterior parts of the DLPFC would be associated with the core processes of cognitive control, supporting the anterior-to-posterior functional organization of the DLPFC.

In our results, the middle-ventral subregions did not show anatomical connections with the IPS but they were functionally coupled with the MDN. One explanation of this discrepancy is that a weak anatomical connection between two regions may still hold a high functional significance via indirect connections of shared brain regions (Friston, 2002; Damoiseaux and Greicius, 2009). Functional connectivity does not necessarily require direct, physical connections and several studies have reported functional connections between regions without anatomical connectivity (Damoiseaux and Greicius, 2009). Therefore, the functional connectivity without physical connections potentially results from indirect anatomical connections via shared brain areas.

The DLPFC is functionally and structurally connected with subcortical areas including the insular and ACC (Catani et al., 2012; Cieslik et al., 2013). As core areas of the MDN, insular and ACC play a role in cognitively demanding tasks, responding to uncertainty and emotional salience (Seeley et al., 2007; Menon and Uddin, 2010; Camilleri et al., 2018). A meta-analysis study demonstrated strong functional connectivity between DLPFC and insular/ACC (Cieslik et al., 2013). We also showed significant functional connectivity between insular/ACC and the DLPFC. However, our tractography analyses showed structural connections between the DLPFC and insular only, not the ACC. With a lower threshold, we found some evidence of a connection between the DLPFC regions and ACC (25\% of participants). In addition, we showed that the corticostriatum projections directly link all DLPFC subregions to the basal ganglia and thalamus (Alexander et al., 1986; Jarbo and Verstynen, 2015). In particular, the anatomical connections between the basal ganglia and DLPFC form a neural circuit involved in several aspects of goal directed behaviours (for a review, see Haber, 2003), which supports a role for the DLPFC in action control (Petrides, 2005b; Cieslik et al., 2013). Furthermore, the extensive connections from the basal ganglia to the cerebral cortex potentially account for the discrepancy between the structural and functional connectivity in the DLPFC subregions.

Although most studies investigating the MDN have focused on the context of goal-directed tasks, it has been shown to play an important role in regulating selfgenerated thoughts, autobiographical memory, social and emotional information, 
which are thought to be associated with the DMN (Spreng and Grady, 2010). In particular, DLPFC may be a key region to play a crucial role in modulating them (Spreng et al., 2018; Turnbull et al., 2019). Emerging evidence suggest that the executive and default network coupling is associated with self-regulation, memory, planning, cognitive control, and creative thinking (Spreng et al., 2010; AndrewsHanna et al., 2014; Gerlach et al., 2014; Spreng et al., 2014; Beaty et al., 2015). Consistent with these studies, our results demonstrated that DLPFC was coupled with both DMN and MD, showing differential connectivity with subregions of DLPFC. Our findings support the default-executive coupling hypothesis that default and control networks can cooperate to facilitate goal-directed cognition (Andrews-Hanna et al., 2014).

In the current study, we explored the structural and functional connectivity across the subregions of DLPFC using probabilistic tractography and rsfMRI approaches. The key limitations of probabilistic tractography are the issues of distance effect and thresholding (Jones, 2008; Morris et al., 2008). A degree of uncertainty in fibre orientation exists at each step in the propagation of a pathway. This accumulation of uncertainty from voxel to voxel as the streamline is advanced causes a decrease in probability with increasing path length and a progressive dispersion of the streamlines with the distance from the seed (Morris et al., 2008). Therefore, it is difficult to determine a threshold value which will identify true positives while simultaneously minimising the rate of both Type I errors in regions close to the seed and Type II errors in distant regions. Although our procedure most likely produced a conservative cut-off value for longer pathways (Binney et al., 2012; Cloutman et al., 2012), there may be long-range connections left undetected.

\section{Author contribution}

J.J: Conceptualization, Methodology, Formal analysis, Investigation, Data curation, Writing - original draft, Writing - review \& editing, Visualization.

M.A.L.R: Methodology, Investigation, Writing - review \& editing.

R.L.J: Methodology, Writing - original draft, Writing - review \& editing. 


\section{References}

Alexander GE, DeLong MR, Strick PL (1986) Parallel organization of functionally segregated circuits linking basal ganglia and cortex. Annu Rev Neurosci 9:357-381.

Alves PN, Foulon C, Karolis V, Bzdok D, Margulies DS, Volle E, Thiebaut de Schotten M (2019) An improved neuroanatomical model of the default-mode network reconciles previous neuroimaging and neuropathological findings. Commun Biol 2:370.

Ashburner J (2007) A fast diffeomorphic image registration algorithm. Neuroimage 38:95-113.

Bahlmann J, Blumenfeld RS, D'Esposito M (2014) The Rostro-Caudal Axis of Frontal Cortex Is Sensitive to the Domain of Stimulus Information. Cereb Cortex.

Bajada CJ, Haroon HA, Azadbakht H, Parker GJ, Lambon Ralph MA, Cloutman LL (2016) The tract terminations in the temporal lobe: Their location and associated functions. Cortex.

Bajada CJ, Jackson RL, Haroon HA, Azadbakht H, Parker GJM, Lambon Ralph MA, Cloutman LL (2017) A graded tractographic parcellation of the temporal lobe. Neuroimage 155:503-512.

Binney RJ, Parker GJ, Lambon Ralph MA (2012) Convergent connectivity and graded specialization in the rostral human temporal lobe as revealed by diffusion-weighted imaging probabilistic tractography. J Cogn Neurosci 24:1998-2014.

Brass M, Derrfuss J, Forstmann B, von Cramon DY (2005) The role of the inferior frontal junction area in cognitive control. Trends Cogn Sci 9:314-316.

Brodmann K (1908) Contributions to the histological localisation of the cerebral cortex VI Announcement The arrangement of the cortex in humans. J Psychol Neurol 10:229-244.

Buckner RL, Andrews-Hanna JR, Schacter DL (2008) The brain's default network - Anatomy, function, and relevance to disease. Ann Ny Acad Sci 1124:1-38.

Camilleri JA, Muller VI, Fox P, Laird AR, Hoffstaedter F, Kalenscher T, Eickhoff SB (2018) Definition and characterization of an extended multiple-demand network. Neuroimage 165:138-147.

Catani M, Dell'Acqua F, Vergani F, Malik F, Hodge H, Roy P, Valabregue R, Thiebaut de Schotten M (2012) Short frontal lobe connections of the human brain. Cortex 48:273-291.

Christoff K, Irving ZC, Fox KC, Spreng RN, Andrews-Hanna JR (2016) Mind-wandering as spontaneous thought: a dynamic framework. Nat Rev Neurosci 17:718-731.

Cieslik EC, Zilles K, Caspers S, Roski C, Kellermann TS, Jakobs O, Langner R, Laird AR, Fox PT, Eickhoff SB (2013) Is There One DLPFC in Cognitive Action Control? Evidence for Heterogeneity From Co-Activation-Based Parcellation. Cereb Cortex 23:2677-2689.

Cloutman LL, Binney RJ, Drakesmith M, Parker GJ, Lambon Ralph MA (2012) The variation of function across the human insula mirrors its patterns of structural connectivity: evidence from in vivo probabilistic tractography. Neuroimage 59:3514-3521.

Damoiseaux JS, Greicius MD (2009) Greater than the sum of its parts: a review of studies combining structural connectivity and resting-state functional connectivity. Brain Structure \& Function 213:525-533.

Duncan J (2010) The multiple-demand (MD) system of the primate brain: mental programs for intelligent behaviour. Trends Cogn Sci 14:172-179.

Duncan J, Owen AM (2000) Common regions of the human frontal lobe recruited by diverse cognitive demands. Trends Neurosci 23:475-483.

Eickhoff SB, Stephan KE, Mohlberg H, Grefkes C, Fink GR, Amunts K, Zilles K (2005) A new SPM toolbox for combining probabilistic cytoarchitectonic maps and functional imaging data. Neuroimage 25:1325-1335.

Embleton KV, Haroon HA, Morris DM, Ralph MA, Parker GJ (2010) Distortion correction for diffusionweighted MRI tractography and fMRI in the temporal lobes. Hum Brain Mapp 31:1570-1587.

Farah MJ, McClelland JL (1991) A computational model of semantic memory impairment: modality specificity and emergent category specificity. J Exp Psychol Gen 120:339-357.

Friston K (2002) Functional integration and inference in the brain. Prog Neurobiol 68:113-143.

Friston KJ, Williams S, Howard R, Frackowiak RS, Turner R (1996) Movement-related effects in fMRI time-series. Magn Reson Med 35:346-355. 
Gottlieb J (2007) From thought to action: the parietal cortex as a bridge between perception, action, and cognition. Neuron 53:9-16.

Haber SN (2003) The primate basal ganglia: parallel and integrative networks. J Chem Neuroanat 26:317-330.

Halai AD, Welbourne SR, Embleton K, Parkes LM (2014) A comparison of dual gradient-echo and spin-echo $\mathrm{fMRI}$ of the inferior temporal lobe. Hum Brain Mapp 35:4118-4128.

Haroon HA, Morris DM, Embleton KV, Alexander DC, Parker GJ (2009) Using the model-based residual bootstrap to quantify uncertainty in fiber orientations from Q-ball analysis. IEEE Trans Med Imaging 28:535-550.

Hoshi E (2006) Functional specialization within the dorsolateral prefrontal cortex: a review of anatomical and physiological studies of non-human primates. Neurosci Res 54:73-84.

Jackson RL, Hoffman P, Pobric G, Lambon Ralph MA (2016) The Semantic Network at Work and Rest: Differential Connectivity of Anterior Temporal Lobe Subregions. J Neurosci 36:1490-1501.

Jackson RL, Bajada CJ, Lambon Ralph MA, Cloutman LL (2020) The Graded Change in Connectivity across the Ventromedial Prefrontal Cortex Reveals Distinct Subregions. Cereb Cortex 30:165180.

Jackson RL, Bajada CJ, Rice GE, Cloutman LL, Lambon Ralph MA (2018) An emergent functional parcellation of the temporal cortex. Neuroimage 170:385-399.

Jarbo K, Verstynen TD (2015) Converging structural and functional connectivity of orbitofrontal, dorsolateral prefrontal, and posterior parietal cortex in the human striatum. J Neurosci 35:3865-3878.

Jeurissen B, Leemans A, Jones DK, Tournier JD, Sijbers J (2011) Probabilistic fiber tracking using the residual bootstrap with constrained spherical deconvolution. Hum Brain Mapp 32:461-479.

Jones DK (2008) Studying connections in the living human brain with diffusion MRI. Cortex 44:936952.

Jung J, Cloutman LL, Binney RJ, Lambon Ralph MA (2017) The structural connectivity of higher order association cortices reflects human functional brain networks. Cortex 97:221-239.

Jung J, Visser M, Binney RJ, Lambon Ralph MA (2018) Establishing the cognitive signature of human brain networks derived from structural and functional connectivity. Brain Struct Funct 223:4023-4038.

Koechlin E, Ody C, Kouneiher F (2003) The architecture of cognitive control in the human prefrontal cortex. Science 302:1181-1185.

Kohn N, Eickhoff SB, Scheller M, Laird AR, Fox PT, Habel U (2014) Neural network of cognitive emotion regulation--an ALE meta-analysis and MACM analysis. Neuroimage 87:345-355.

Kriegeskorte N, Kievit RA (2013) Representational geometry: integrating cognition, computation, and the brain. Trends Cogn Sci 17:401-412.

Kriegeskorte N, Mur M, Bandettini P (2008) Representational similarity analysis - connecting the branches of systems neuroscience. Front Syst Neurosci 2:4.

Maldjian JA, Laurienti PJ, Kraft RA, Burdette JH (2003) An automated method for neuroanatomic and cytoarchitectonic atlas-based interrogation of fMRI data sets. Neuroimage 19:1233-1239.

Martino J, Brogna C, Robles SG, Vergani F, Duffau H (2010) Anatomic dissection of the inferior fronto-occipital fasciculus revisited in the lights of brain stimulation data. Cortex 46:691-699.

Menon V, Uddin LQ (2010) Saliency, switching, attention and control: a network model of insula function. Brain Structure \& Function 214:655-667.

Miller BL, Cummings JL (2007) The human frontal lobes : functions and disorders, 2nd Edition. New York: Guilford Press.

Moayedi M, Salomons TV, Dunlop KA, Downar J, Davis KD (2015) Connectivity-based parcellation of the human frontal polar cortex. Brain Struct Funct 220:2603-2616.

Morecraft RJ, Cipolloni PB, Stilwell-Morecraft KS, Gedney MT, Pandya DN (2004) Cytoarchitecture and cortical connections of the posterior cingulate and adjacent somatosensory fields in the rhesus monkey. J Comp Neurol 469:37-69. 
Morris DM, Embleton KV, Parker GJ (2008) Probabilistic fibre tracking: differentiation of connections from chance events. Neuroimage 42:1329-1339.

Morris R, Pandya DN, Petrides M (1999) Fiber system linking the mid-dorsolateral frontal cortex with the retrosplenial/presubicular region in the rhesus monkey. J Comp Neurol 407:183-192.

Nee DE, Wager TD, Jonides J (2007) Interference resolution: insights from a meta-analysis of neuroimaging tasks. Cogn Affect Behav Neurosci 7:1-17.

Parker GJ, Alexander DC (2005) Probabilistic anatomical connectivity derived from the microscopic persistent angular structure of cerebral tissue. Philos Trans R Soc Lond B Biol Sci 360:893902.

Parker GJ, Luzzi S, Alexander DC, Wheeler-Kingshott CA, Ciccarelli O, Lambon Ralph MA (2005) Lateralization of ventral and dorsal auditory-language pathways in the human brain. Neuroimage 24:656-666.

Petrides M (1985) Deficits in non-spatial conditional associative learning after periarcuate lesions in the monkey. Behav Brain Res 16:95-101.

Petrides M (2005a) The rostral-caudal axis of cognitive control within the lateral frontal cortex. In From monkey brain to human brain. Cambridge: MA: MIT Press.

Petrides M (2005b) Lateral prefrontal cortex: architectonic and functional organization. Philos T Roy Soc B 360:781-795.

Petrides M, Pandya DN (1984) Projections to the frontal cortex from the posterior parietal region in the rhesus monkey. J Comp Neurol 228:105-116.

Petrides M, Pandya DN (1999) Dorsolateral prefrontal cortex: comparative cytoarchitectonic analysis in the human and the macaque brain and corticocortical connection patterns. Eur J Neurosci 11:1011-1036.

Poser BA, Versluis MJ, Hoogduin JM, Norris DG (2006) BOLD contrast sensitivity enhancement and artifact reduction with multiecho EPI: parallel-acquired inhomogeneity-desensitized fMRI. Magn Reson Med 55:1227-1235.

Raichle ME, MacLeod AM, Snyder AZ, Powers WJ, Gusnard DA, Shulman GL (2001) A default mode of brain function. Proc Natl Acad Sci U S A 98:676-682.

Ramnani N, Owen AM (2004) Anterior prefrontal cortex: insights into function from anatomy and neuroimaging. Nat Rev Neurosci 5:184-194.

Rottschy C, Langner R, Dogan I, Reetz K, Laird AR, Schulz JB, Fox PT, Eickhoff SB (2012) Modelling neural correlates of working memory: a coordinate-based meta-analysis. Neuroimage 60:830-846.

Schmahmann JD, Pandya DN, Wang R, Dai G, D'Arceuil HE, de Crespigny AJ, Wedeen VJ (2007) Association fibre pathways of the brain: parallel observations from diffusion spectrum imaging and autoradiography. Brain 130:630-653.

Seeley WW, Menon V, Schatzberg AF, Keller J, Glover GH, Kenna H, Reiss AL, Greicius MD (2007) Dissociable intrinsic connectivity networks for salience processing and executive control. J Neurosci 27:2349-2356.

Sormaz M, Murphy C, Wang HT, Hymers M, Karapanagiotidis T, Poerio G, Margulies DS, Jefferies E, Smallwood J (2018) Default mode network can support the level of detail in experience during active task states. Proc Natl Acad Sci U S A 115:9318-9323.

Spreng RN, Sepulcre J, Turner GR, Stevens WD, Schacter DL (2013) Intrinsic Architecture Underlying the Relations among the Default, Dorsal Attention, and Frontoparietal Control Networks of the Human Brain. J Cognitive Neurosci 25:74-86.

Thiebaut de Schotten M, Dell'Acqua F, Valabregue R, Catani M (2012) Monkey to human comparative anatomy of the frontal lobe association tracts. Cortex 48:82-96.

Tournier JD, Yeh CH, Calamante F, Cho KH, Connelly A, Lin CP (2008) Resolving crossing fibres using constrained spherical deconvolution: validation using diffusion-weighted imaging phantom data. Neuroimage 42:617-625. 
762

763

764

765

766

767

768

769

770

Walker AE (1940) A cytoarchitectural study of the prefrontal area of the macaque monkey. J Comp Neurol 73:59-86.

Woolgar A, Hampshire A, Thompson R, Duncan J (2011) Adaptive coding of task-relevant information in human frontoparietal cortex. J Neurosci 31:14592-14599.

Yeterian EH, Pandya DN, Tomaiuolo F, Petrides M (2012) The cortical connectivity of the prefrontal cortex in the monkey brain. Cortex 48:58-81.

Zheng D, Oka T, Bokura H, Yamaguchi S (2008) The key locus of common response inhibition network for no-go and stop signals. J Cogn Neurosci 20:1434-1442. 
A

ค B

2

c

(1)

$\sum$

0

đ

$+$

은

(1)

0

0

$<$

"ত

0

B

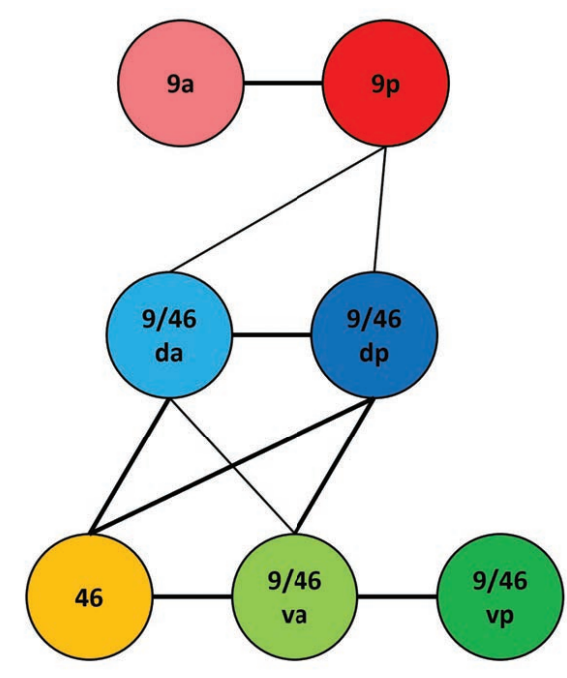

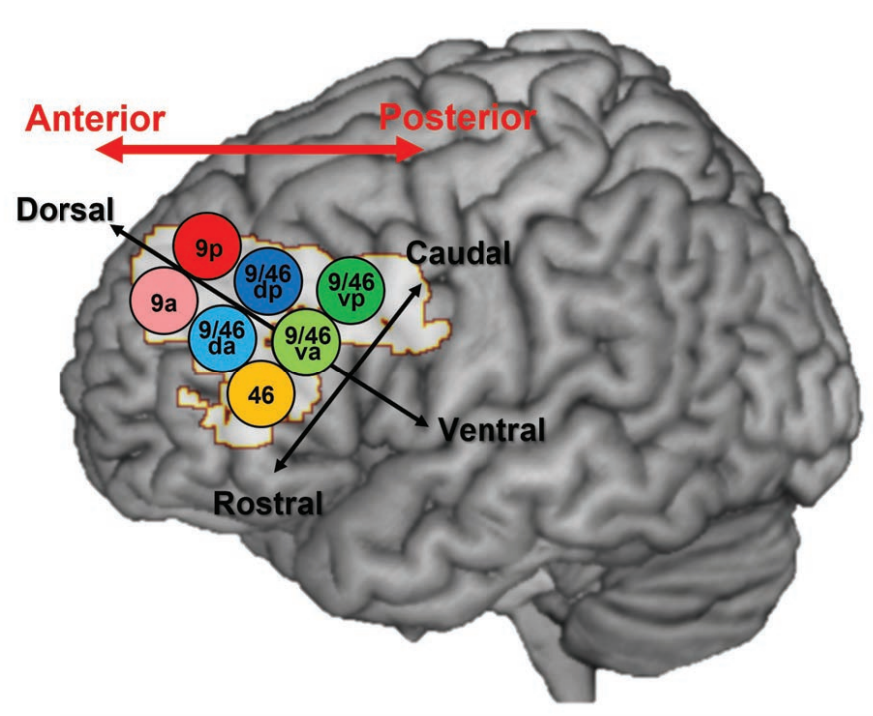

$\square$ DLPFC (BA9 + BA46)

C

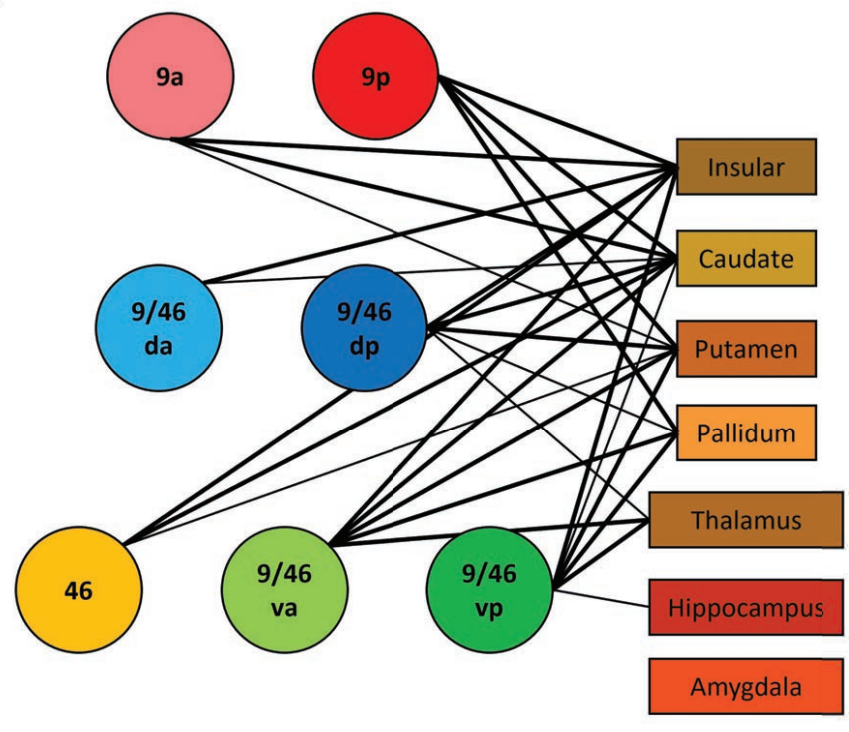

D

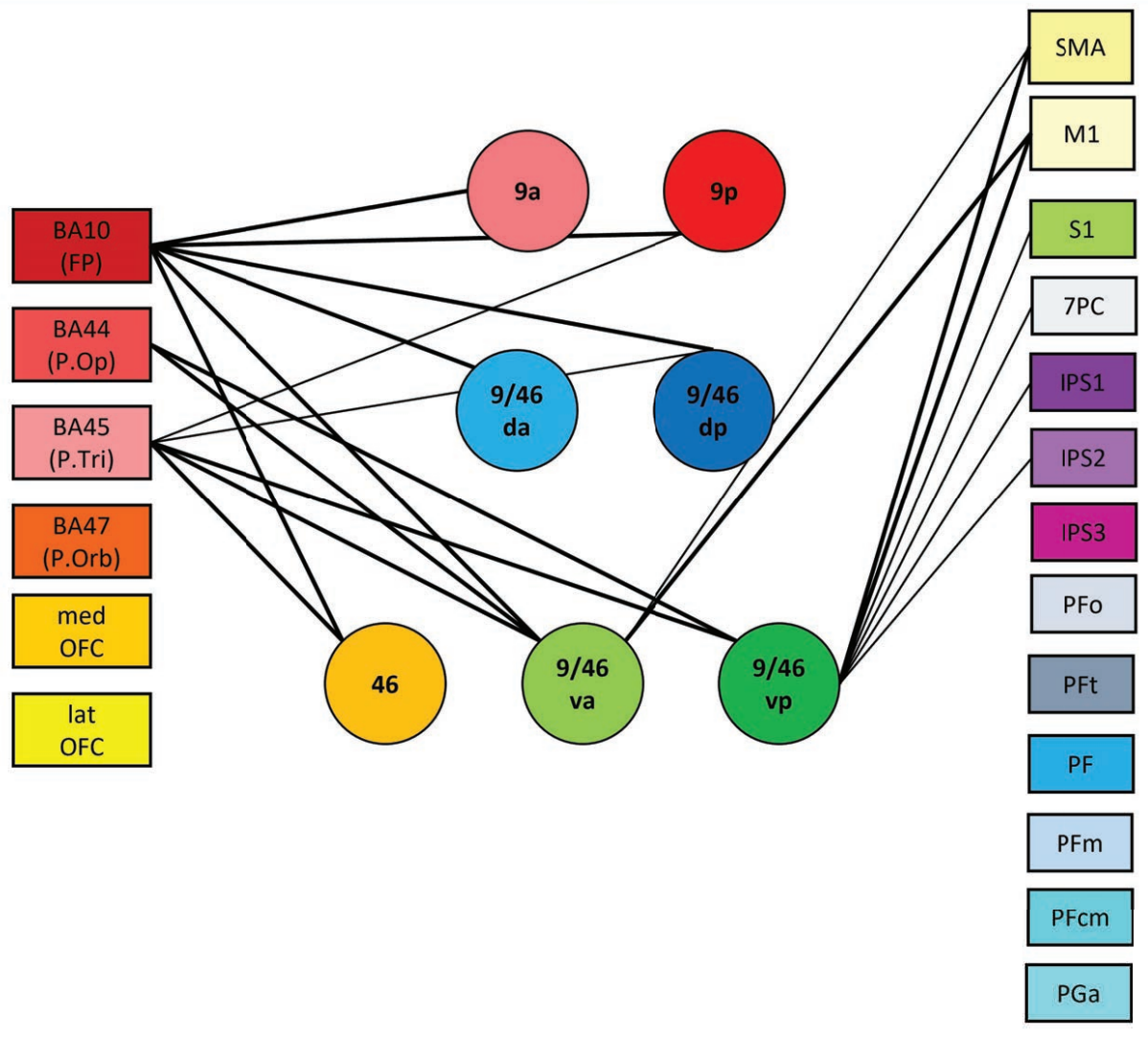


LEFT

RIGHT
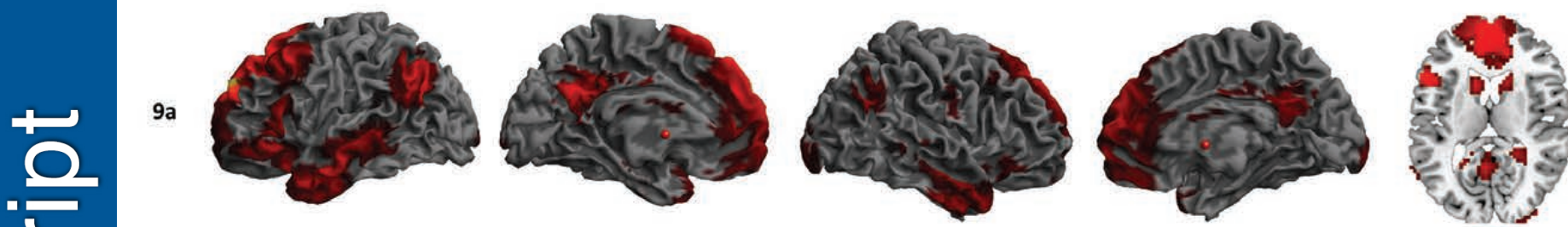

0

0
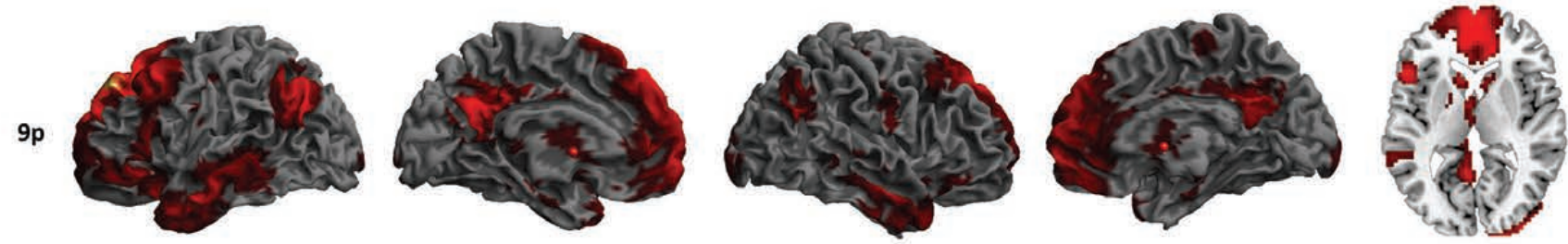

$\sum$
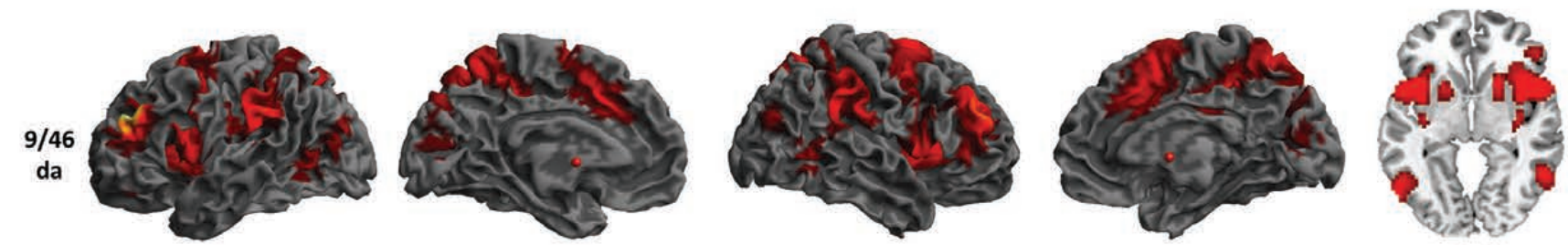

(1)

$+$

(1)

$9 / 46$
$d p$
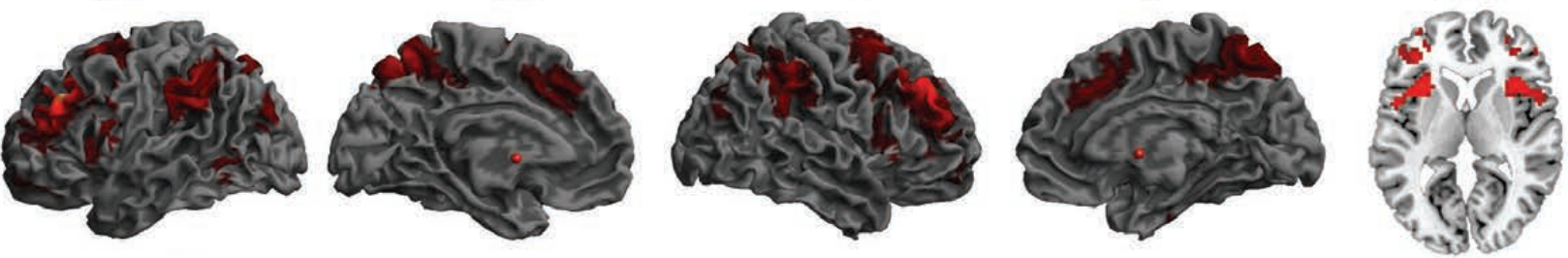

4
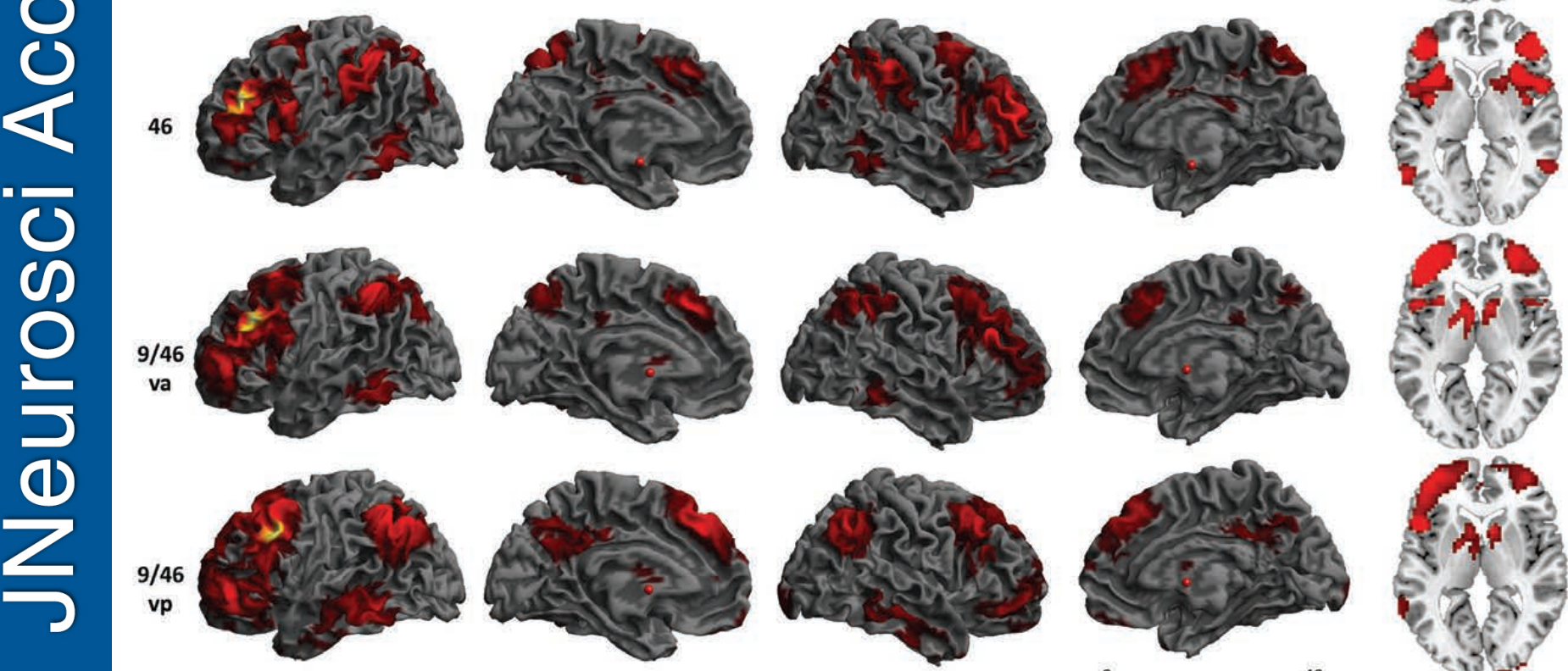

0

${ }^{40}$ t value

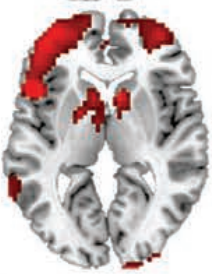


RIGHT
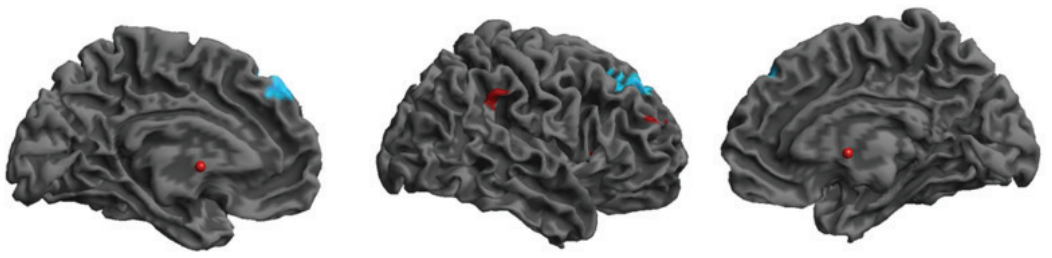

9/46da vs.
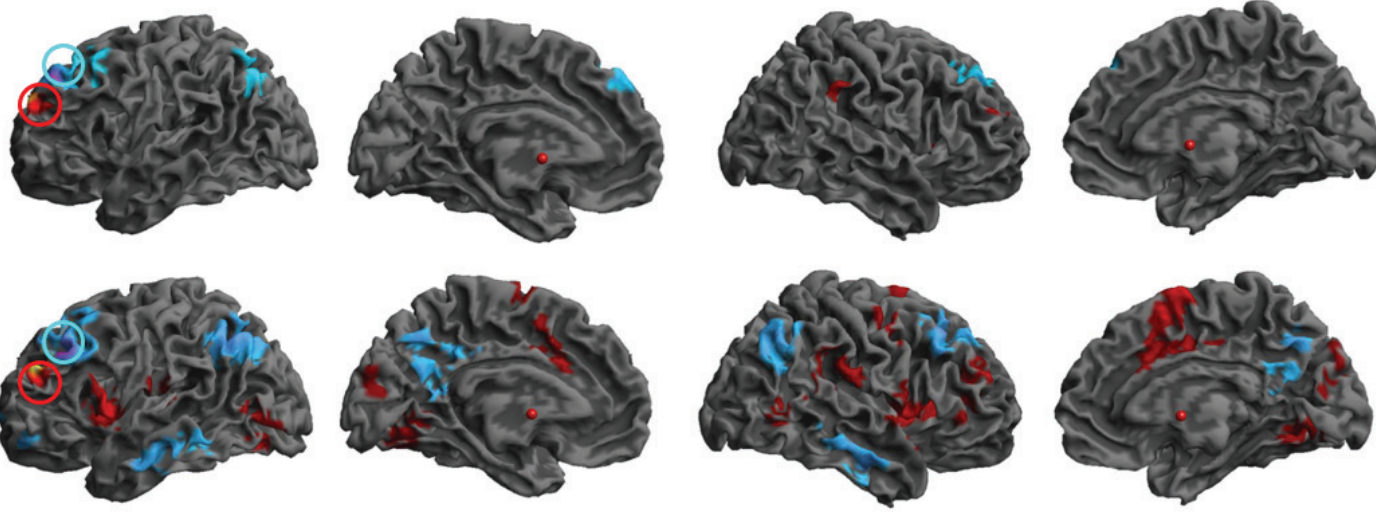

46 vs.
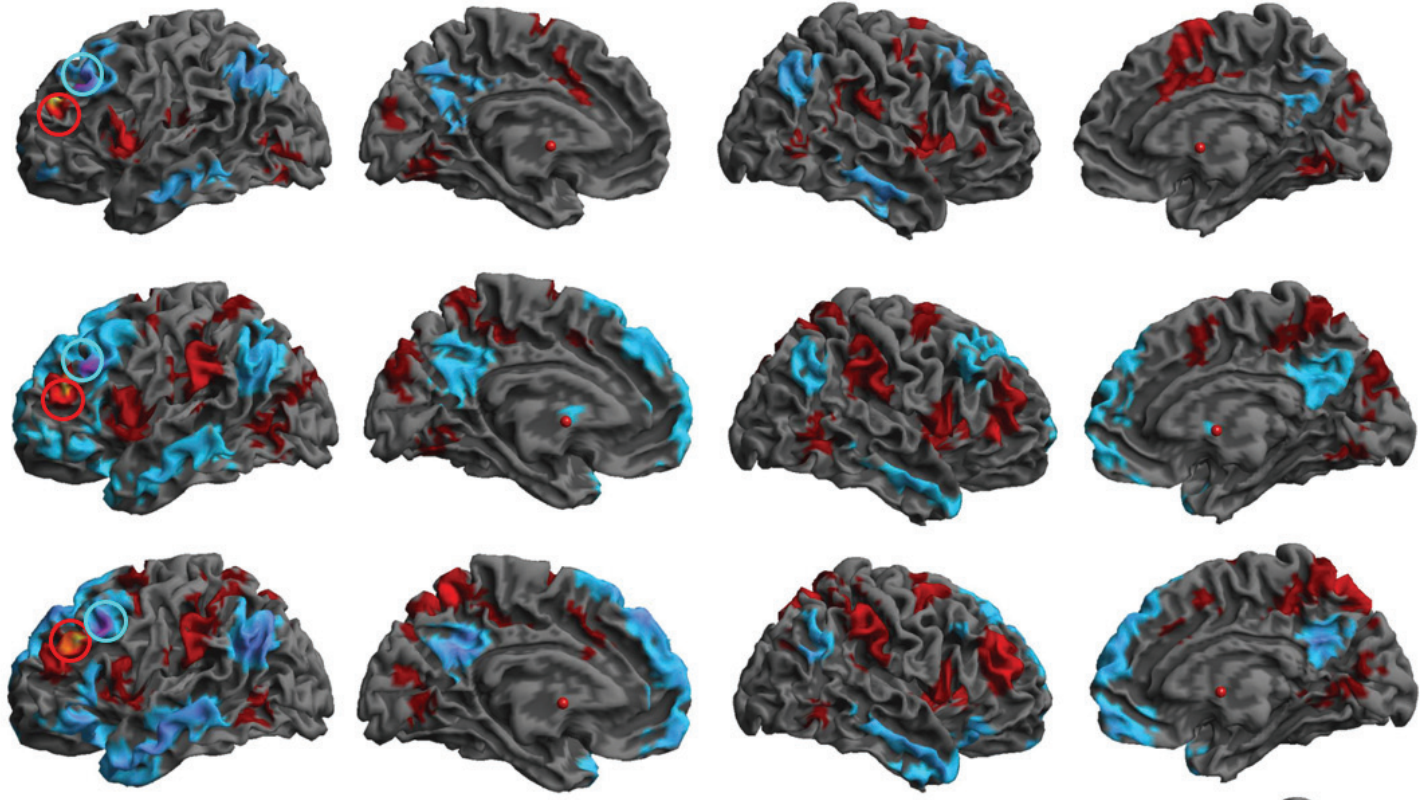

9/46va vs.
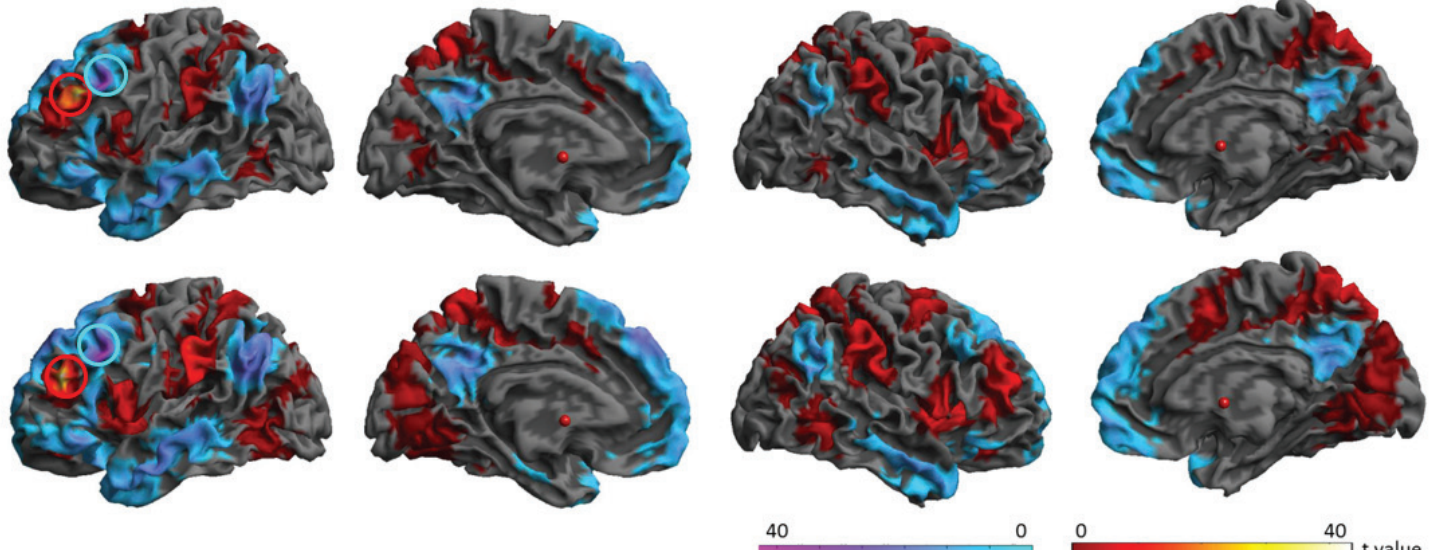

40 t value 
RIGHT

Dorsal vs. Middle
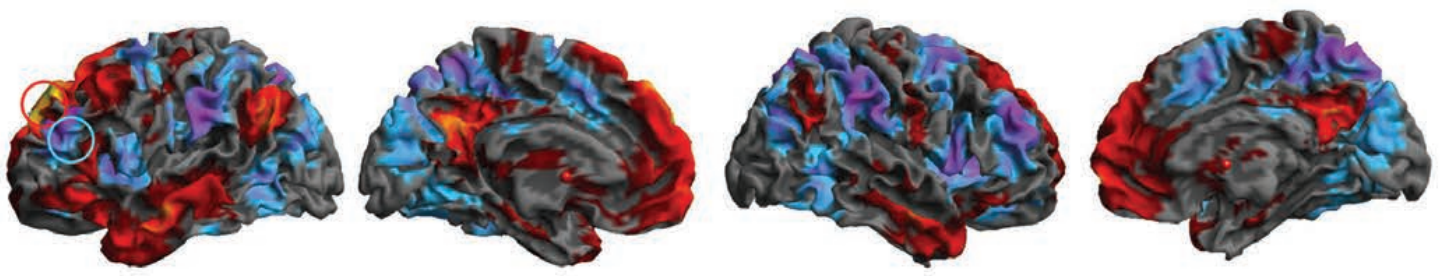

Middle vs. Ventral
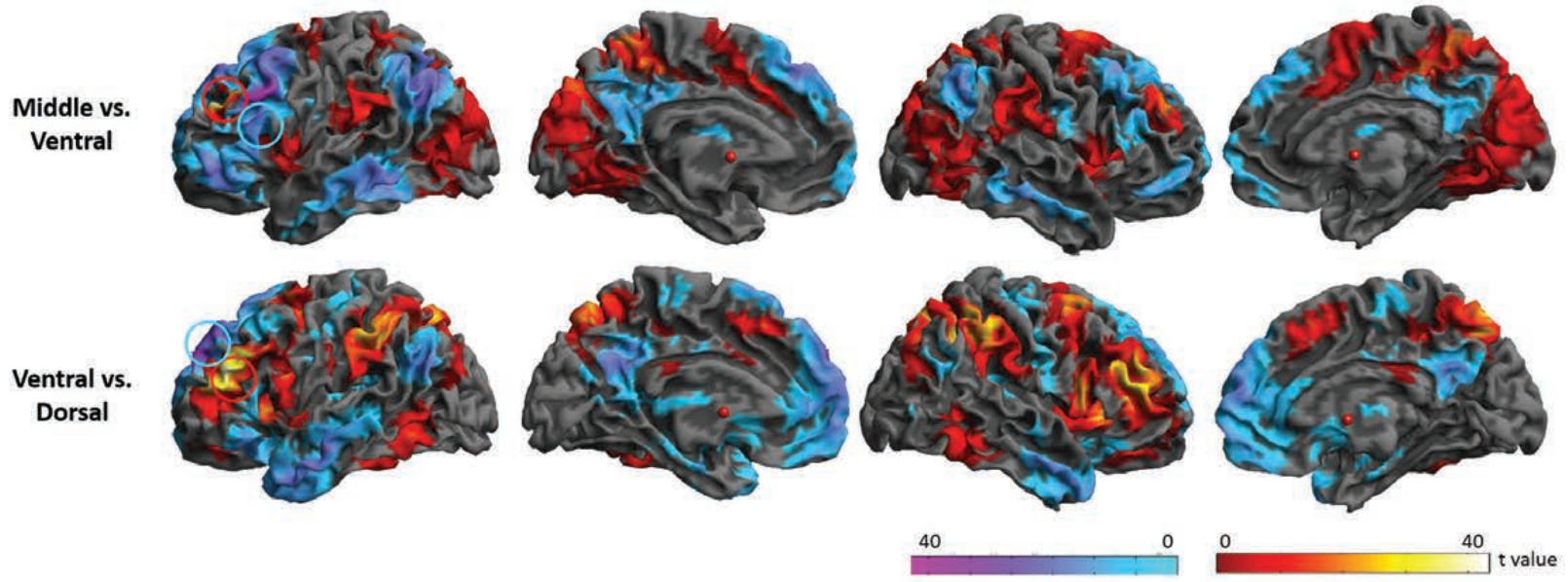
A

The results of structural connectivity patterns

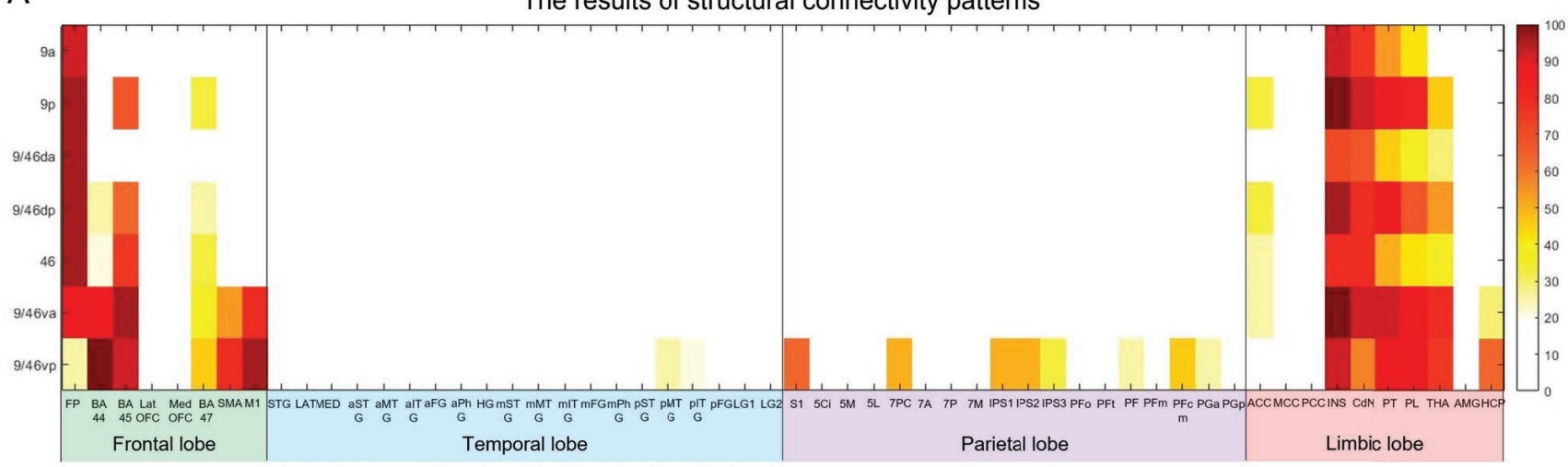

The results of functional connectivity patterns

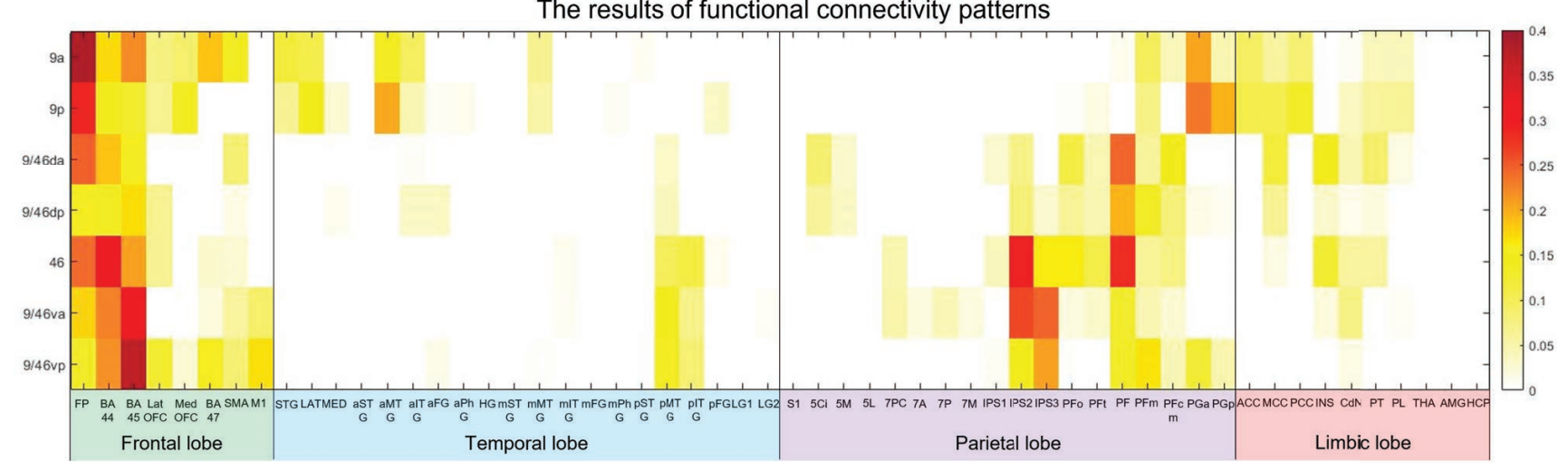

B
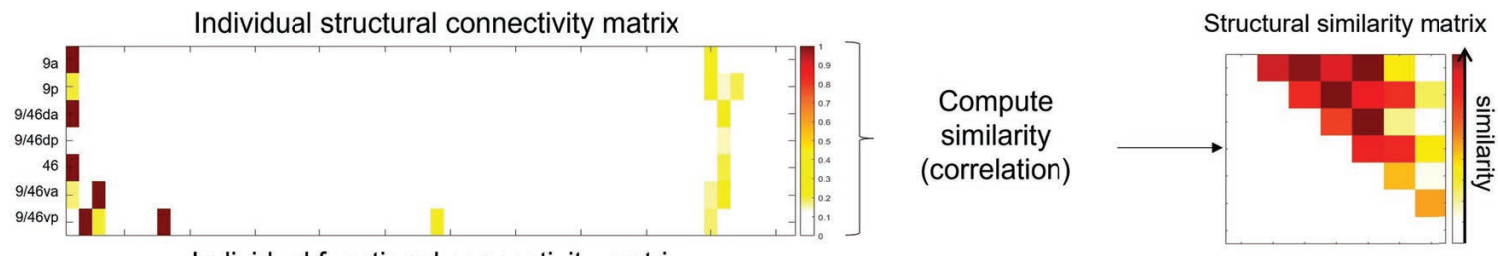

Individual functional connectivity matrix
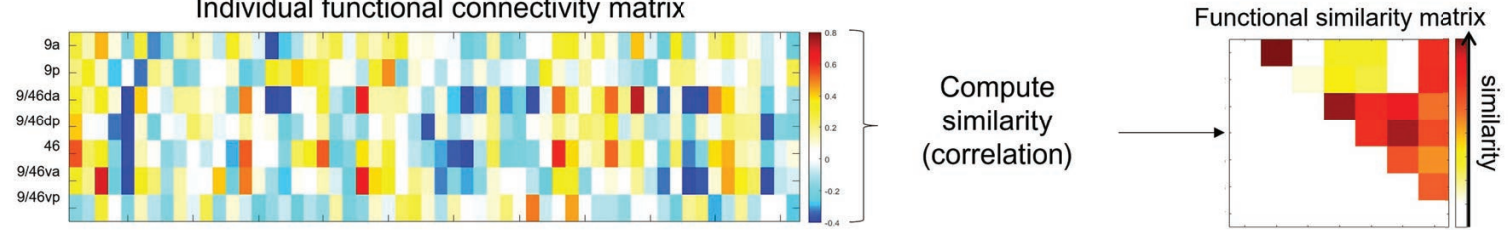

C

The similarity results of structural connectivity patterns

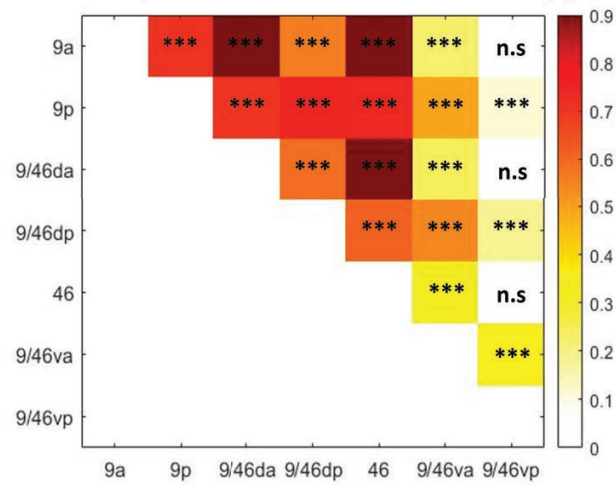

The similarity results of functional connectivity patterns

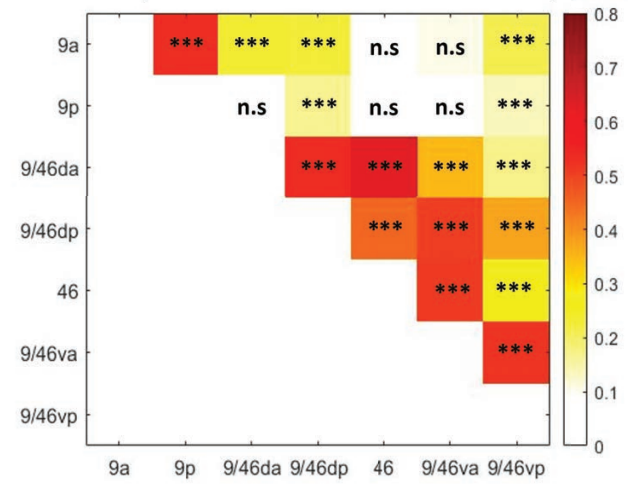

